\title{
Decentralized Channel Decisions of Green Supply Chain in a Fuzzy Decision Making Environment
}

\author{
Shengju Sang* \\ Department of Economics and Management, Heze University, \\ No.2269 University Road, Heze, Shandong, 274015, China ${ }^{\dagger}$ \\ E-mail: sangshengju@163.com
}

Received 20 November 2016

Accepted 19 June 2017

\begin{abstract}
This paper considers the greening policies in a decentralized channel between one manufacturer and one retailer in a fuzzy decision making environment. We consider the manufacturing cost and the parameters of demand function as the fuzzy variables. Based on the different market structures, we develop three different fuzzy decentralized decision models. For each case, the expected value, optimistic value and pessimistic value models are formulated, and their optimal solutions are also derived through the fuzzy set theory. Finally, three numerical examples are solved to examine the effectiveness of fuzzy models. The effects of the confidence level of the supply chain member's profits and the fuzziness of parameters on optimal prices, level of green innovation, and fuzzy expected profits of actors are also analyzed.
\end{abstract}

Keywords: Green supply chain, Game theory, Fuzzy theory, Fuzzy variables

\section{Introduction}

In a traditional supply chain, chain members usually focus on their total costs and profits, and ignore their operations impacted on environment. This phenomenon is altering rapidly as the problems of environment are affecting the population's living conditions of the world more severely. With the development of the green economy and low-carbon economy, more and more scholars and market administrators have applied the green principles and techniques to develop and solve the green supply chain (GSC) management problems.

Recently, some studies have been done on analyzing the game theoretic models in GSC management. Sheu ${ }^{1}$ considered the nuclear power generation problem by using a multi-objective optimization programming approach in GSC management. Using the method of asymmetrical Nash bargaining game and backward induction, Sheu ${ }^{2}$ analyzed the negotiations problem with government intervention between the GSC members. Ghosh and $\mathrm{Shah}^{3}$ developed a game theoretic model for analyzing the influence of channel structures on greening levels, prices and profits in both cooperation and noncooperation situations. Ghosh and $\mathrm{Shan}^{4}$ also discussed the coordination issues of GMC by using the cost sharing contract. By using evolutionary dynamics, Barari et al. ${ }^{5}$ framed integrated and holistic conceptual framework on maximizing the total supply chain's profits that took the practical aspects into account in a GSC. Swami and Shah ${ }^{6}$ established a two-part tariff contract for coordinating GSC, where the models contained price and non-price variables. Similar issues was studied by Swami and $\mathrm{Shah}^{7}$, they studied the coordination mechanism of GSC that took the shelf-space allocation of products into

\footnotetext{
* Department of Economics and Management, Heze University, Heze, Shandong, China

${ }^{\dagger}$ China, Heze University, E-mail: sangshengju@163.com
} 
consideration. Mirzapour Al-e-hashem ${ }^{8}$ proposed a stochastic programming model to investigate the production-distribution planning system after demand fluctuation in a GSC. Tomasin et al. ${ }^{9}$ used multiple case studies to investigate the elements that contribute to increase sales of green products in Brazil. Zhang and $\mathrm{Liu}^{10}$ proposed three types of coordination mechanisms to coordinate the three stage green supply chain.

The works mentioned above discussed the game theoretic models of green supply chain with deterministic market demands and production costs. However, in real world, there exist many in deterministic factors for some new green products. The production costs and market demands of these green products are often lack of historical data. Moreover, with the market uncertainty, the exact data are derived more and more unavailable due to the short of product life cycles. Thus, the fuzzy set theory, instead of probability theory is fit well for dealing with greening policy problem. In recent years, fuzzy sets theory is used by many researches to deal with the modes of supply chain. For instance, $\mathrm{Xu}$ and $\mathrm{Zhai}{ }^{11,12}$ presented fuzzy newsboy models in which the market demands were fuzzy. Zhou et al. ${ }^{13}$ investigated the operations between one-manufacturer and one-retailer, where the customer's demand was fuzziness. Yu and $\mathrm{Jin}^{14}$ studied the decentralized channel, integrated channel and return contract models with fuzzy retail prices and demands. Wei and $Z^{2}{ }^{15}$ proposed fuzzy models with pricing competition of two retailers in a closed-loop supply chain. Ye and $\mathrm{Li}^{16}$ investigated the Stackelberg models in which the demand was fuzzy. In addition, Zhao et al. ${ }^{17}$ studied the pricing competitive strategies in which one manufacturer sold his substitutable products to two competing retailers with fuzzy costs and demands. Zhao et al. ${ }^{18}$ also studied pricing competition problems with two manufacturers under fuzzy demand environments. Wei and $\mathrm{Zhao}^{19}$ discussed the problems of pricing decisions in a reverse channel. Zhao and Wang ${ }^{20}$ studied service and price competition problem with two competitive retailers in a distribution system with fuzzy demand. Yu et al. ${ }^{21}$ developed fuzzy newsboy models to obtain the optimal prices between the supplier and the retailer. Recently, Sang ${ }^{22}$ investigated the coordination mechanism in a multiple supply chain with fuzzy demands and costs. Chang and $\mathrm{Yeh}^{23}$ analyzed the decentralized and the centralized supply chain system with fuzzy demand under a return policy, and showed that the fuzziness of demand affected the optimal results of the supply chain members. Khamseh et al. ${ }^{24}$ proposed four different pricing models of complementary products with two competing manufacturers in fuzzy environments Sang $^{25}$ proposed one expected value model and two chance-constrained programming models between two competing manufacturers and one common retailer under fuzzy uncertainty.

To the best of our knowledge, there is no work that deals with the optimal decisions of GSC in a fuzzy decision environment. Therefore, in this paper, we will examine how the retailer and the manufacturer should make their own pricing and green level decisions in a fuzzy decision environment. We also explore the impacts of the confidence level and the fuzzy degree of parameters on the equilibrium prices, green level and profits of the supply chain members.

The paper makes three contributions to the extant literature. Firstly, our proposed models extend the study of Ghosh and $\mathrm{Shah}^{3}$ by considering the greening policies under fuzzy uncertainty. The manufacturing costs and market demand are all fuzzy. Secondly, we apply the different ranking measures of fuzzy variables to reflect the attitudes of the participants. They can choose the expected values model to derive their optimal decisions if they are risk neutral. They can choose the optimistic values model to derive their optimal decisions if they are risk preferable. And they can choose the pessimistic values model to derive their optimal decisions if they are risk averse. Thirdly, compared to the method used in conventional environment, our work has some main findings: the profit margin, wholesale price, retail price and level of green innovation are higher, while the manufacturer's profit is lower, and the retailer's profit and supply chain system's profit are higher under fuzzy uncertainly.

The rest of this paper is as follows. Firstly, some useful concepts and propositions about fuzzy set theory are presented in Section 2. Section 3 introduces the notations of the models. Three fuzzy green supply chain models are proposed in Section 4. In Section 5, three numerical examples are given to elucidate the solutions of each model. Section 6 summarizes the work.

\section{Preliminaries}

The possibility measure Pos was introduced by Nahmias $^{26}$ and three axioms were given:

Axiom 1. $\operatorname{Pos}(\Theta)=1$, for the nonempty set $\Theta$. 
Axiom 2. $\operatorname{Pos}(\phi)=0$, for the empty set $\phi$.

Axiom 3. $\operatorname{Pos}\left(\bigcup_{i=1}^{m} A_{i}\right)=\sup _{1 \leq i \leq m} \operatorname{Pos}\left(A_{i}\right)$, for any events $A_{i}$ in $P(\Theta)$, where $P$ denotes the power set of $\Theta$.

Definition 1(Liu and $\mathrm{Liu}^{27}$ ). If $\operatorname{Pos}\{\xi \leq 0\}=0$, then the fuzzy variable $\xi$ is called positive.

Definition 2 (Liu and $\mathrm{Liu}^{27}$ ). Let $\xi$ be a fuzzy variable. Then, for any $\alpha \in(0,1], \xi_{\alpha}^{R}=\sup \{r \mid \operatorname{Pos}\{\xi \geq r\} \geq \alpha\}$ and $\xi_{\alpha}^{L}=\inf \{r \mid \operatorname{Pos}\{\xi \leq r\} \geq \alpha\}$ are said to be the $\alpha$ optimistic value and the $\alpha$-pessimistic value of $\xi$, respectively.

Example 1. Let $\xi$ be a triangular fuzzy variable with $\xi=(a, b, c)$, then $\xi_{\alpha}^{R}$ and $\xi_{\alpha}^{L}$ are given as

$$
\xi_{\alpha}^{R}=\alpha b+(1-\alpha) c \text { and } \xi_{\alpha}^{L}=\alpha b+(1-\alpha) a .
$$

Lemma 1 (Liu and $\mathrm{Liu}^{27}$ and Zhao et al. ${ }^{28}$ ). Let $\xi$ and $\eta$ be positive independent fuzzy variables. Then, for any $\alpha \in(0,1]$
(a) $(\xi+\eta)_{\alpha}^{R}=\xi_{\alpha}^{R}+\eta_{\alpha}^{R}$ and $(\xi+\eta)_{\alpha}^{L}=\xi_{\alpha}^{L}+\eta_{\alpha}^{L}$;
(b) $(\xi \cdot \eta)_{\alpha}^{R}=\xi_{\alpha}^{R} \cdot \eta_{\alpha}^{R}$ and $(\xi \cdot \eta)_{\alpha}^{L}=\xi_{\alpha}^{L} \cdot \eta_{\alpha}^{L}$;
(c) $(\xi-\eta)_{\alpha}^{R}=\xi_{\alpha}^{R}-\eta_{\alpha}^{L}$ and $(\xi-\eta)_{\alpha}^{L}=\xi_{\alpha}^{L}-\eta_{\alpha}^{R}$.

Lemma 2. (Liu and $\mathrm{Liu}^{27}$ ). Let $\xi$ be a fuzzy variable with finite expected value, then

$$
E[\xi]=\frac{1}{2} \int_{0}^{1}\left(\xi_{\alpha}^{L}+\xi_{\alpha}^{R}\right) \mathrm{d} \alpha .
$$

Lemma 3 (Liu and $\mathrm{Liu}^{27}$ ). Let $\xi$ and $\eta$ be independent fuzzy variables with finite expected values. Then for any numbers $m$ and $n$

$$
E[m \xi+n \eta]=m E[\xi]+n E[\eta] .
$$

Definition 2. Let $\xi$ and $\eta$ be positive independent fuzzy variables, $\xi>\eta$ if and only if for any $\alpha \in(0,1]$, $\xi_{\alpha}^{L}>\eta_{\alpha}^{L}$ and $\xi_{\alpha}^{R}>\eta_{\alpha}^{R}$

Definition 3. Let $\xi$ and $\eta$ be positive independent fuzzy variables, if $\xi>\eta$, then $E[\xi]>E[\eta]$.

\section{Problem Descriptions}

Consider a GSC with a manufacturer and a retailer, where the retailer orders greening products from the manufacturer, and then he retails them to end customers. The manufacturer is assumed to produce only one product and the retailer sells only single product.
Similar to Ghosh and $\mathrm{Shah}^{3}$, the market demand function faced by the manufacturer and the retailer is considered as a linear form of the retail price $p$ and the level of greening innovation $\theta$, the market demand is

$$
\tilde{q}=\tilde{D}-\tilde{\beta} p+\tilde{\gamma} \theta .
$$

where the fuzzy parameter $\tilde{D}$ denotes the market potential, the fuzzy parameter $\tilde{\beta}$ denotes the retail price sensitivity of the customer, and the fuzzy parameter $\tilde{\gamma}$ denotes the greening innovation sensitivity of the manufacturer to the demand. The fuzzy parameters $\tilde{D}, \tilde{\beta}$ and $\tilde{\gamma}$ are positive and mutually independent.

Further, let $w$ denote the unit wholesale price of greening product, $\tilde{c}$ the unit fuzzy producing cost of greening product for the manufacturer and $m$ the unit margin profit of product for the retailer. As the retail price $p$ is the sum of margin profit $m$ and wholesale price $w$, we consider retail price as $p=m+w$. The fuzzy demand function of the greening product is presented as follows

$$
\tilde{q}=\tilde{D}-\tilde{\beta}(m+w)+\tilde{\gamma} \theta .
$$

It is assumed that the marginal cost of the manufacturer is not affected by the greening innovation. In addition, for achieving greening innovation, it needs fixed investment. The cost of the fixed investment is assumed as a quadratic function of the parameter $\theta$, and is expressed as $\tilde{I} \theta^{2}$, where $\tilde{I}$ denotes fuzzy fixed investment coefficient. The demand is positive in the real world. Thus we have $\operatorname{Pos}\{\tilde{D}-\tilde{\beta}(m+w)+\tilde{\gamma} \theta<0\}=0$, where Pos is a possibility measure. The order quantity of the greening product can be presented as $q=E[\tilde{q}]$.

The fuzzy profit functions of the manufacturer and the retailer can be derived as

$$
\begin{aligned}
& \Pi_{M}=(w-\tilde{c})(\tilde{D}-\tilde{\beta}(m+w)+\tilde{\gamma} \theta)-\tilde{I} \theta^{2} . \\
& \Pi_{R}=m(\tilde{D}-\tilde{\beta}(m+w)+\tilde{\gamma} \theta) .
\end{aligned}
$$

\section{Model Analysis}

In this section, we will discuss the operations of the supply chain participants in a GSC, and examine the manufacturer and the retailer how to set their optimal policies with different power structures under fuzzy uncertainty. We study the equilibrium decisions under three non-cooperative games in the decentralized channel: the manufacturer dominates the channel (Manufacturer-Stackelberg game), the retailer leads the 
channel (Retailer-Stackelberg game), and the supply chain participants have an equal bargaining power (Vertical-Nash game).

\subsection{Manufacturer Stackelberg (MS) game}

In the MS game, the retailer has less bargaining power than the manufacturer. That is to say, the manufacturer is the leader in the GSC. Firstly, the manufacturer sets his wholesale price $w$ and level of greening innovation $\theta$ condition on the retailer's optimal reaction to his decisions. Then, the retailer sets is own margin profit $m$. Hence, in this case, we can formulate the fuzzy optimal model as follows

$$
\left\{\begin{array}{l}
\max _{w, \theta} E\left[\Pi_{M}\left(w, \theta, m^{*}\right)\right]=E\left[(w-\tilde{c})(\tilde{D}-\tilde{\beta}(m+w)+\tilde{\gamma} \theta)-\tilde{I} \theta^{2}\right] \\
\begin{array}{l}
\text { Pos }\{w-\tilde{c}<0\}=0 \\
\text { where } m^{*} \text { derives from problem: }
\end{array} \\
\qquad\left\{\begin{array}{l}
\max _{m} E\left[\Pi_{R}(m)\right]=E[m(\tilde{D}-\tilde{\beta}(m+w)+\tilde{\gamma} \theta)] \\
\text { s.t. } \\
\operatorname{Pos}\{\tilde{D}-\tilde{\beta}(m+w)+\tilde{\gamma} \theta<0\}=0
\end{array}\right.
\end{array}\right.
$$

where $E\left[\Pi_{M}\left(w, \theta, m^{*}\right)\right]$ and $E\left[\Pi_{R}(m)\right]$ represent the fuzzy expected profit of the manufacturer and the retailer.

We first solve the reaction function of the retailer.

Theorem 1. If $\operatorname{Pos}\left\{\tilde{D}-\tilde{\beta}\left(m^{*}+w\right)+\tilde{\gamma} \theta<0\right\}=0$, then the retailer's optimal reaction function is given as

$$
m^{*}(w, \theta)=\frac{E[\tilde{D}]-E[\tilde{\beta}] w+E[\tilde{\gamma}] \theta}{2 E[\tilde{\beta}]} .
$$

Proof. The fuzzy expected profit $E\left[\Pi_{R}(m)\right]$ is

$$
E\left[\Pi_{R}(m)\right]=-E[\tilde{\beta}] m^{2}+(E[\tilde{D}]-E[\tilde{\beta}] w+E[\tilde{\gamma}] \theta) m
$$

The first order condition

$$
\frac{\mathrm{d}}{\mathrm{d} m} E\left[\Pi_{R}(m)\right]=-2 E[\tilde{\beta}] m+E[\tilde{D}]-E[\tilde{\beta}] w+E[\tilde{\gamma}] \theta .
$$

The second order condition

$$
\frac{\mathrm{d}^{2}}{\mathrm{~d} m^{2}} E\left[\Pi_{R}(m)\right]=-2 E[\tilde{\beta}]<0 .
$$

Thus, the retailer's fuzzy expected profit $E\left[\Pi_{R}(m)\right]$ is concave in $m$.
Let the first order condition be zero, the result (6) can be derived.

Theorem 1 is proved.

Here, we consider $m^{*}(w, \theta)$ as the optimal response to the decisions of the manufacturer. Having the follower's information about the decision, the leader sets his optimal decisions. So, the following Proposition can be derived.

Theorem 2. If $(E[\tilde{\gamma}])^{2}<8 E[\tilde{\beta}] E[\tilde{I}], \operatorname{Pos}\left\{w^{*}-\tilde{c}<0\right\}=0$ and $\operatorname{Pos}\left\{\tilde{D}-\tilde{\beta}\left(m^{*}+w^{*}\right)+\tilde{\gamma} \theta^{*}<0\right\}=0$, then the optimal equilibrium decisions are

$$
\begin{aligned}
& m^{*}= \frac{E[\tilde{\gamma}]\left(2 E[\tilde{\beta} \tilde{c}] E[\tilde{\gamma}]-E[\tilde{\beta}] \int_{0}^{1}\left(\tilde{\gamma}_{\alpha}^{L} \tilde{c}_{\alpha}^{R}+\tilde{\gamma}_{\alpha}^{R} \tilde{c}_{\alpha}^{L}\right) \mathrm{d} \alpha\right)}{2 E[\tilde{\beta}]\left(8 E[\tilde{\beta}] E[\tilde{I}]-(E[\tilde{\gamma}])^{2}\right)} \\
&+\frac{2 E[\tilde{I}](E[\tilde{D}]-E[\tilde{\beta} \tilde{c}])}{8 E[\tilde{\beta}] E[\tilde{I}]-(E[\tilde{\gamma}])^{2}} . \\
& w^{*}=\frac{E[\tilde{\gamma}]\left(E[\tilde{\beta} \tilde{c}] E[\tilde{\gamma}]-E[\tilde{\beta}] \int_{0}^{1}\left(\tilde{\gamma}_{\alpha}^{L} \tilde{c}_{\alpha}^{R}+\tilde{\gamma}_{\alpha}^{R} \tilde{c}_{\alpha}^{L}\right) \mathrm{d} \alpha\right)}{E[\tilde{\beta}]\left(8 E[\tilde{\beta}] E[\tilde{I}]-(E[\tilde{\gamma}])^{2}\right)} \\
&+\frac{4 E[\tilde{I}](E[\tilde{D}]+E[\tilde{\beta} \tilde{c}])}{8 E[\tilde{\beta}] E[\tilde{I}]-(E[\tilde{\gamma}])^{2}} .
\end{aligned}
$$

$$
\theta^{*}=\frac{E[\tilde{\gamma}](E[\tilde{D}]+3 E[\tilde{\beta} \tilde{c}])-2 E[\tilde{\beta}] \int_{0}^{1}\left(\tilde{\gamma}_{\alpha}^{L} \tilde{c}_{\alpha}^{R}+\tilde{\gamma}_{\alpha}^{R} \tilde{c}_{\alpha}^{L}\right) \mathrm{d} \alpha}{8 E[\tilde{\beta}] E[\tilde{I}]-(E[\tilde{\gamma}])^{2}}
$$

Proof. The fuzzy expected profit $E\left[\Pi_{M}\right]$ is

$$
\begin{aligned}
E\left[\Pi_{M}\right]= & \frac{1}{2} \int_{0}^{1}\left((w-\tilde{c})(\tilde{D}-\tilde{\beta}(m+w)+\tilde{\gamma} \theta)-\tilde{I} \theta^{2}\right)_{\alpha}^{L} \mathrm{~d} \alpha \\
& +\frac{1}{2} \int_{0}^{1}\left((w-\tilde{c})(\tilde{D}-\tilde{\beta}(m+w)+\tilde{\gamma} \theta)-\tilde{I} \theta^{2}\right)_{\alpha}^{R} \mathrm{~d} \alpha \\
= & -E[\tilde{\beta}] w^{2}-E[\tilde{I}] \theta^{2}+E[\tilde{\gamma}] w \theta \\
& +(E[\tilde{D}]+E[\tilde{\beta} \tilde{c}]-E[\tilde{\beta}] m) w \\
& -\frac{1}{2} \theta \int_{0}^{1}\left(\tilde{\gamma}_{\alpha}^{L} \tilde{c}_{\alpha}^{R}+\tilde{\gamma}_{\alpha}^{R} \tilde{c}_{\alpha}^{L}\right) \mathrm{d} \alpha+E[\tilde{\beta} \tilde{c}] m \\
& -\frac{1}{2} \int_{0}^{1}\left(\tilde{D}_{\alpha}^{L} \tilde{c}_{\alpha}^{R}+\tilde{D}_{\alpha}^{R} \tilde{c}_{\alpha}^{L}\right) \mathrm{d} \alpha
\end{aligned}
$$

Substituting $m$ in Eq.(6) into Eq.(11), the first order conditions can be obtained as 


$$
\begin{aligned}
\frac{\partial}{\partial w} E\left[\Pi_{M}\right]= & -E[\tilde{\beta}] w+\frac{1}{2} E[\tilde{\gamma}] \theta+\frac{1}{2}(E[\tilde{D}]+E[\tilde{\beta} \tilde{c}]) . \\
\frac{\partial}{\partial \theta} E\left[\Pi_{M}\right]= & -2 E[\tilde{I}] \theta+\frac{1}{2} E[\tilde{\gamma}] w+\frac{E[\tilde{\beta} \tilde{c}] E[\tilde{\gamma}]}{2 E[\tilde{\beta}]} \\
& -\frac{1}{2} \int_{0}^{1}\left(\tilde{\gamma}_{\alpha}^{L} \tilde{c}_{\alpha}^{R}+\tilde{\gamma}_{\alpha}^{R} \tilde{c}_{\alpha}^{L}\right) \mathrm{d} \alpha .
\end{aligned}
$$

Therefore, the Hessian matrix of $E\left[\Pi_{M}\right]$ is

$$
\mathrm{H}=\left[\begin{array}{cc}
-E[\tilde{\beta}] & \frac{1}{2} E[\tilde{\gamma}] \\
\frac{1}{2} E[\tilde{\gamma}] & -2 E[\tilde{I}]
\end{array}\right] .
$$

Since $E[\tilde{\beta}]>0, E[\tilde{I}]>0$ and $(E[\tilde{\gamma}])^{2}<8 E[\tilde{\beta}] E[\tilde{I}]$, thus $\mathrm{H}$ is a definite negative matrix and $E\left[\Pi_{M}\right]$ is jointly concave in $w$ and $\theta$.

Let the first order conditions be zero, we can have Eqs.(9) and (10).

Substituting $w^{*}$ in Eq. (9) and $\theta^{*}$ in Eq. (10) into Eq.(6), we can get Eq.(8).

Theorem 2 is proved.

The chance-constrained programming proposed by Liu and Iwamura ${ }^{29,30}$, has a big role in formulating fuzzy models. Given a confidence level $\alpha$, the decision makers try to optimize the critical value subject to chance constraint. Hence, in this case, we can formulate the maximax chance-constrained programming model as follows

$$
\left\{\begin{array}{l}
\max _{w, \theta} \bar{\Pi}_{M} \\
\text { s.t. } \\
\operatorname{Pos}\left\{(w-\tilde{c})\left(\tilde{D}-\tilde{\beta}\left(m^{*}+w\right)+\tilde{\gamma} \theta\right)-\tilde{I} \theta^{2} \geq \bar{\Pi}_{M}\right\} \geq \alpha \\
\operatorname{Pos}\{w-\tilde{c}<0\}=0 \\
\text { where } m^{*} \text { derives from problem: } \\
\qquad \begin{array}{l}
\max _{m} \bar{\Pi}_{R} \\
\text { s.t. } \\
\operatorname{Pos}\left\{m(\tilde{D}-\tilde{\beta}(m+w)+\tilde{\gamma} \theta) \geq \bar{\Pi}_{R}\right\} \geq \alpha \\
\operatorname{Pos}\{\tilde{D}-\tilde{\beta}(m+w)+\tilde{\gamma} \theta<0\}=0
\end{array}
\end{array}\right.
$$

where $\alpha$ is a given confidence level for manufacturer's and retailer's profits, $\bar{\Pi}_{R}$ is the maximum value that $\Pi_{R}(m)$ obtains with at least possibility $\alpha$, and $\bar{\Pi}_{M}$ is the maximum value that $\Pi_{M}\left(w, \theta, m^{*}\right)$ obtains with at least possibility $\alpha$. Therefore, model (12) equals to the following model (13), in which supply chain actors attempt to maximize the $\alpha$-optimistic value of profits
$\left(\Pi_{M}\left(w, \theta, m^{*}\right)\right)_{\alpha}^{R}$ and $\left(\Pi_{R}(m)\right)_{\alpha}^{R}$ by choosing the optimal solutions, that is

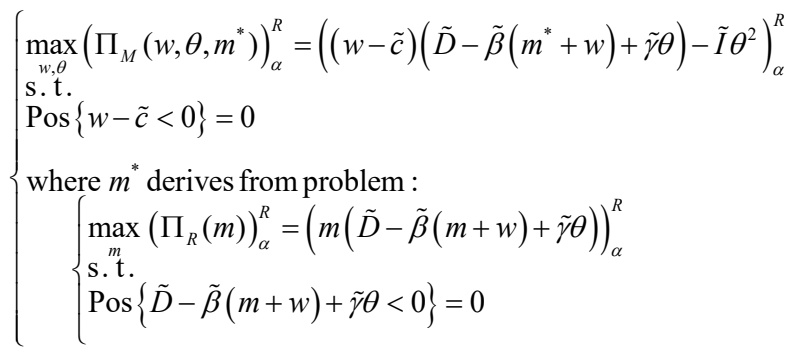

Theorem 3. Let $\left(\Pi_{R}(m)\right)_{\alpha}^{R}$ be the retailer's $\alpha$-optimistic value of profit. The wholesale price $w$ and the level of greening innovation $\theta$ set by manufacturer are fixed. If $\operatorname{Pos}\left\{\tilde{D}-\tilde{\beta}\left(m^{*}+w\right)+\tilde{\gamma} \theta<0\right\}=0$, then optimal reaction function is given as

$$
m^{*}(w, \theta)=\frac{\tilde{D}_{\alpha}^{R}-\tilde{\beta}_{\alpha}^{L} w+\tilde{\gamma}_{\alpha}^{R} \theta}{2 \tilde{\beta}_{\alpha}^{L}} .
$$

Proof. The retailer's profit is

$$
\left(\Pi_{R}(m)\right)_{\alpha}^{R}=-\tilde{\beta}_{\alpha}^{L} m^{2}+\left(\tilde{D}_{\alpha}^{R}-\tilde{\beta}_{\alpha}^{L} w+\tilde{\gamma}_{\alpha}^{R} \theta\right) m
$$

The first order condition

$$
\frac{\mathrm{d}}{\mathrm{d} m}\left(\Pi_{R}(m)\right)_{\alpha}^{R}=-2 \tilde{\beta}_{\alpha}^{L} m+\tilde{D}_{\alpha}^{R}-\tilde{\beta}_{\alpha}^{L} w+\tilde{\gamma}_{\alpha}^{R} \theta .
$$

The second order condition

$$
\frac{\mathrm{d}^{2}}{\mathrm{~d} m^{2}}\left(\Pi_{R}(m)\right)_{\alpha}^{R}=-2 \tilde{\beta}_{\alpha}^{L}<0 .
$$

Thus, for any $\alpha \in(0,1],\left(\Pi_{R}(m)\right)_{\alpha}^{R}$ is concave in $m$.

Let $\frac{\mathrm{d}}{\mathrm{d} m}\left(\Pi_{R}(m)\right)_{\alpha}^{R}=0$, we can get the retailer's optimal margin profit as in Eq. (14).

Theorem 3 is proved.

Theorem 4. Let $\left(\Pi_{M}\left(w, \theta, m^{*}\right)\right)_{\alpha}^{R}$ be the manufacturer's $\alpha$-optimistic value of profit. If $\left(\tilde{\gamma}_{\alpha}^{R}\right)^{2}<8 \tilde{\beta}_{\alpha}^{L} \tilde{I}_{\alpha}^{L}$, $\operatorname{Pos}\left\{\tilde{D}-\tilde{\beta}\left(m^{*}+w^{*}\right)+\tilde{\gamma} \theta^{*}<0\right\}=0$ and $\operatorname{Pos}\left\{w^{*}-\tilde{c}<0\right\}=0$,

then the optimal equilibrium decisions are

$$
m^{*}=\frac{2 \tilde{I}_{\alpha}^{L}\left(\tilde{D}_{\alpha}^{R}-\tilde{\beta}_{\alpha}^{L} \tilde{c}_{\alpha}^{L}\right)}{8 \tilde{\beta}_{\alpha}^{L} \tilde{I}_{\alpha}^{L}-\left(\tilde{\gamma}_{\alpha}^{R}\right)^{2}} .
$$




$$
\begin{aligned}
& w^{*}=\frac{4 \tilde{I}_{\alpha}^{L}\left(\tilde{D}_{\alpha}^{R}+\tilde{\beta}_{\alpha}^{L} \tilde{c}_{\alpha}^{L}\right)-\left(\tilde{\gamma}_{\alpha}^{R}\right)^{2} \tilde{c}_{\alpha}^{L}}{8 \tilde{\beta}_{\alpha}^{L} \tilde{I}_{\alpha}^{L}-\left(\tilde{\gamma}_{\alpha}^{R}\right)^{2}} . \\
& \theta^{*}=\frac{\tilde{\gamma}_{\alpha}^{R}\left(\tilde{D}_{\alpha}^{R}-\tilde{\beta}_{\alpha}^{L} \tilde{c}_{\alpha}^{L}\right)}{8 \tilde{\beta}_{\alpha}^{L} \tilde{I}_{\alpha}^{L}-\left(\tilde{\gamma}_{\alpha}^{R}\right)^{2}} .
\end{aligned}
$$

Proof. The manufacturer's profit is

$$
\begin{gathered}
\left(\Pi_{M}\left(w, \theta, m^{*}\right)\right)_{\alpha}^{R}=\left((w-\tilde{c})\left(\tilde{D}-\tilde{\beta}\left(m^{*}+w\right)+\tilde{\gamma} \theta\right)-\tilde{I} \theta^{2}\right)_{\alpha}^{R} \\
=\left(w-\tilde{c}_{\alpha}^{L}\right)\left(\tilde{D}_{\alpha}^{R}-\tilde{\beta}_{\alpha}^{L}\left(m^{*}+w\right)+\tilde{\gamma}_{\alpha}^{R} \theta\right)-\tilde{I}_{\alpha}^{L} \theta^{2} .
\end{gathered}
$$

Substituting $m^{*}(w, \theta)$ in Eq. (14) into Eq. (19), the first order conditions can be obtained as

$$
\begin{aligned}
& \frac{\partial}{\partial w}\left(\Pi_{M}\left(w, \theta, m^{*}\right)\right)_{\alpha}^{R}=-\tilde{\beta}_{\alpha}^{L} w+\frac{1}{2} \tilde{\gamma}_{\alpha}^{R} \theta+\frac{1}{2}\left(\tilde{D}_{\alpha}^{R}+\tilde{\beta}_{\alpha}^{L} \tilde{c}_{\alpha}^{L}\right) . \\
& \frac{\partial}{\partial \theta}\left(\Pi_{M}\left(w, \theta, m^{*}\right)\right)_{\alpha}^{R}=-2 \tilde{I}_{\alpha}^{L} \theta+\frac{1}{2} \tilde{\gamma}_{\alpha}^{R} w-\frac{1}{2} \tilde{\gamma}_{\alpha}^{R} \tilde{c}_{\alpha}^{L} .
\end{aligned}
$$

Therefore, the Hessian matrix of $\left(\Pi_{M}\left(w, \theta, m^{*}\right)\right)_{\alpha}^{R}$ is

$$
\mathrm{H}=\left[\begin{array}{cc}
-\tilde{\beta}_{\alpha}^{L} & \frac{1}{2} \tilde{\gamma}_{\alpha}^{R} \\
\frac{1}{2} \tilde{\gamma}_{\alpha}^{R} & -2 \tilde{I}_{\alpha}^{L}
\end{array}\right] .
$$

Since $\tilde{\beta}, \tilde{I}$ are positive fuzzy variables and $\left(\tilde{\gamma}_{\alpha}^{R}\right)^{2}<8 \tilde{\beta}_{\alpha}^{L} \tilde{I}_{\alpha}^{L}$, thus, for any $\alpha \in(0,1], \mathrm{H}$ is a definite negative matrix and $\left(\Pi_{M}\right)_{\alpha}^{R}$ is jointly concave in $w$ and $\theta$.

Let the first order conditions be zero, we can have Eqs.(16) and (17).

Substituting $w^{*}$ in Eq. (16) and $\theta^{*}$ in Eq. (17) into Eq.(14), we can get Eq.(18).

Theorem 4 is proved.

The optimal optimistic profits of the manufacturer and the retailer can be easily obtained as follows

$$
\begin{aligned}
&\left(\Pi_{M}\right)_{\alpha}^{R}=\frac{\tilde{I}_{\alpha}^{L}\left(\tilde{D}_{\alpha}^{R}-\tilde{\beta}_{\alpha}^{L} \tilde{c}_{\alpha}^{L}\right)^{2}}{8 \tilde{\beta}_{\alpha}^{L} \tilde{I}_{\alpha}^{L}-\left(\tilde{\gamma}_{\alpha}^{R}\right)^{2}} . \\
&\left(\Pi_{R}\right)_{\alpha}^{R}=\frac{4 \tilde{\beta}_{\alpha}^{L}\left(\tilde{I}_{\alpha}^{L}\right)^{2}\left(\tilde{D}_{\alpha}^{R}-\tilde{\beta}_{\alpha}^{L} \tilde{c}_{\alpha}^{L}\right)^{2}}{\left(8 \tilde{\beta}_{\alpha}^{L} \tilde{I}_{\alpha}^{L}-\left(\tilde{\gamma}_{\alpha}^{R}\right)^{2}\right)^{2}} .
\end{aligned}
$$

Similarly, in the MS game, we can formulate the minimax chance-constrained programming model of GSC as follows

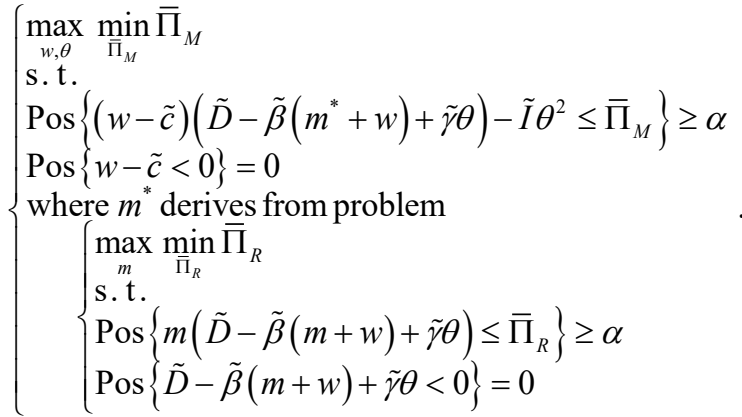

where $\alpha$ is a given confidence level for manufacturer's and retailer's profits, $\bar{\Pi}_{R}$ is the maximum value that $\Pi_{R}(m)$ obtains with at least possibility $\alpha$, and $\bar{\Pi}_{M}$ is the maximum value that $\Pi_{M}\left(w, \theta, m^{*}\right)$ obtains with at least possibility $\alpha$. Therefore, model (20) equals to the following model (21), in which supply chain actors attempt to maximize the $\alpha$-pessimistic value of profits $\left(\Pi_{M}\left(w, \theta, m^{*}\right)\right)_{\alpha}^{L}$ and $\left(\Pi_{R}(m)\right)_{\alpha}^{L}$ by choosing optimal solutions, that is

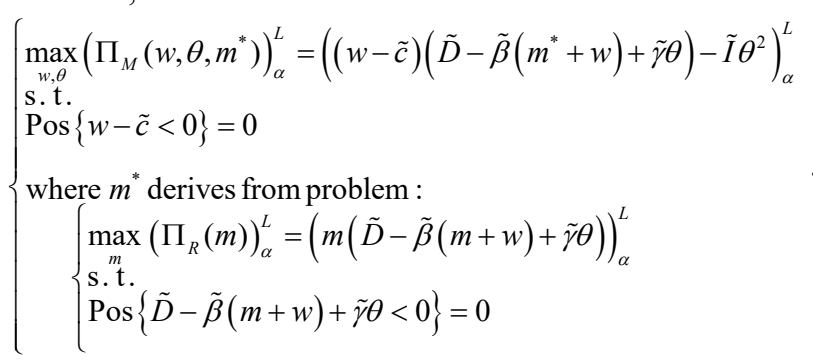

Theorem 5. Let $\left(\Pi_{R}(m)\right)_{\alpha}^{L}$ and $\left(\Pi_{M}\left(w, \theta, m^{*}\right)\right)_{\alpha}^{L}$ be the $\alpha$ pessimistic value of the profits for the retailer and the manufacturer. If $\left(\tilde{\gamma}_{\alpha}^{L}\right)^{2}<8 \tilde{\beta}_{\alpha}^{R} \tilde{I}_{\alpha}^{R}, \quad \operatorname{Pos}\left\{w^{*}-\tilde{c}<0\right\}=0$ and $\operatorname{Pos}\left\{\tilde{D}-\tilde{\beta}\left(m^{*}+w^{*}\right)+\tilde{\gamma} \theta^{*}<0\right\}=0$, then the optimal equilibrium decisions in the MS case are

$$
\begin{aligned}
& m^{*}=\frac{2 \tilde{I}_{\alpha}^{R}\left(\tilde{D}_{\alpha}^{L}-\tilde{\beta}_{\alpha}^{R} \tilde{c}_{\alpha}^{R}\right)}{8 \tilde{\beta}_{\alpha}^{R} \tilde{I}_{\alpha}^{R}-\left(\tilde{\gamma}_{\alpha}^{L}\right)^{2}} . \\
& w^{*}=\frac{4 \tilde{I}_{\alpha}^{R}\left(\tilde{D}_{\alpha}^{L}+\tilde{\beta}_{\alpha}^{R} \tilde{c}_{\alpha}^{R}\right)-\left(\tilde{\gamma}_{\alpha}^{L}\right)^{2} \tilde{c}_{\alpha}^{R}}{8 \tilde{\beta}_{\alpha}^{R} \tilde{I}_{\alpha}^{R}-\left(\tilde{\gamma}_{\alpha}^{L}\right)^{2}} . \\
& \theta^{*}=\frac{\tilde{\gamma}_{\alpha}^{L}\left(\tilde{D}_{\alpha}^{L}-\tilde{\beta}_{\alpha}^{R} \tilde{c}_{\alpha}^{R}\right)}{8 \tilde{\beta}_{\alpha}^{R} \tilde{I}_{\alpha}^{R}-\left(\tilde{\gamma}_{\alpha}^{L}\right)^{2}} .
\end{aligned}
$$

Proof. Similar to Theorems 3 and 4 .

The optimal pessimistic profits of the manufacturer and the retailer are given by 


$$
\begin{aligned}
\left(\Pi_{M}\right)_{\alpha}^{L} & =\frac{\tilde{I}_{\alpha}^{R}\left(\tilde{D}_{\alpha}^{L}-\tilde{\beta}_{\alpha}^{R} \tilde{c}_{\alpha}^{R}\right)^{2}}{8 \tilde{\beta}_{\alpha}^{R} \tilde{I}_{\alpha}^{R}-\left(\tilde{\gamma}_{\alpha}^{L}\right)^{2}} . \\
\left(\Pi_{R}\right)_{\alpha}^{L} & =\frac{4 \tilde{\beta}_{\alpha}^{R}\left(\tilde{I}_{\alpha}^{R}\right)^{2}\left(\tilde{D}_{\alpha}^{L}-\tilde{\beta}_{\alpha}^{R} \tilde{c}_{\alpha}^{R}\right)^{2}}{\left(8 \tilde{\beta}_{\alpha}^{R} \tilde{I}_{\alpha}^{R}-\left(\tilde{\gamma}_{\alpha}^{L}\right)^{2}\right)^{2}} .
\end{aligned}
$$

Remark 1. If $\alpha=1$, it is clear the parameters $\tilde{c}, \tilde{D}$, $\tilde{\beta}, \tilde{\gamma}$ and $\tilde{I}$ reduce to crisp numbers, then the optimal solutions in Theorems 4 and 5 are

$$
\begin{aligned}
m^{*} & =\frac{2 I(D-\beta c)}{8 \beta I-\gamma^{2}} . \\
w^{*} & =\frac{4 I(D+\beta c)-\gamma^{2} c}{8 \beta I-\gamma^{2}} . \\
\theta^{*} & =\frac{\gamma(D-\beta c)}{8 \beta I-\gamma^{2}} .
\end{aligned}
$$

\subsection{Retailer Stackelberg (RS) game}

In the RS game, the manufacturer has more bargaining power than the retailer. That is to say, the retailer is the leader in the GSC. In this case, firstly, the retailer sets his margin profit $m$ condition on the manufacturer's optimal reaction to his decisions. Then, the manufacturer sets his wholesale price $w$ and level of greening innovation $\theta$. Hence, in this case, we can formulate the fuzzy optimal model as follows

$$
\begin{aligned}
& \max _{m} E\left[\Pi_{R}\left(m, w^{* *}, \theta^{* *}\right)\right]=E[m(\tilde{D}-\tilde{\beta}(m+w)+\tilde{\gamma} \theta)] \\
& \text { s.t. } \\
& \text { where } w^{* *} \text { and } \theta^{* *} \text { derive from problem: } \\
& \int \max _{w, \theta} E\left[\Pi_{M}(w, \theta)\right]=E\left[(w-\tilde{c})(\tilde{D}-\tilde{\beta}(m+w)+\tilde{\gamma} \theta)-\tilde{I} \theta^{2}\right] . \\
& \text { s.t. } \\
& \{\operatorname{Pos}\{w-\tilde{c}<0\}=0 \\
& \operatorname{Pos}\{\tilde{D}-\tilde{\beta}(m+w)+\tilde{\gamma} \theta<0\}=0
\end{aligned}
$$

We first solve the reaction function of the manufacturer.

Theorem 6. Let $E\left[\Pi_{M}(w, \theta)\right]$ be the manufacturer's fuzzy expected profit. If $(E[\tilde{\gamma}])^{2}<4 E[\tilde{\beta}] E[\tilde{I}]$, $\operatorname{Pos}\left\{\tilde{D}-\tilde{\beta}\left(m+w^{* *}\right)+\tilde{\gamma} \theta^{* *}<0\right\}=0$ and $\operatorname{Pos}\left\{w^{* *}-\tilde{c}<0\right\}=0$, then the manufacturer's optimal reaction functions are

$$
w^{* *}(m)=\frac{4 E[\tilde{I}](E[\tilde{D}]+E[\tilde{\beta} \tilde{c}])}{2\left(4 E[\tilde{\beta}] E[\tilde{I}]-(E[\tilde{\gamma}])^{2}\right)}
$$

$$
\begin{gathered}
-\frac{E[\tilde{\gamma}] \int_{0}^{1}\left(\tilde{\gamma}_{\alpha}^{L} \tilde{c}_{\alpha}^{R}+\tilde{\gamma}_{\alpha}^{R} \tilde{c}_{\alpha}^{L}\right) \mathrm{d} \alpha+4 E[\tilde{\beta}] E[\tilde{I}] m}{2\left(4 E[\tilde{\beta}] E[\tilde{I}]-(E[\tilde{\gamma}])^{2}\right)} . \\
\theta^{* *}(m)=\frac{E[\tilde{\gamma}](E[\tilde{D}]+E[\tilde{\beta} \tilde{c}])}{4 E[\tilde{\beta}] E[\tilde{I}]-(E[\tilde{\gamma}])^{2}} \\
-\frac{E[\tilde{\beta}] \int_{0}^{1}\left(\tilde{\gamma}_{\alpha}^{L} \tilde{c}_{\alpha}^{R}+\tilde{\gamma}_{\alpha}^{R} \tilde{c}_{\alpha}^{L}\right) \mathrm{d} \alpha+E[\tilde{\beta}] E[\tilde{\gamma}] m}{4 E[\tilde{\beta}] E[\tilde{I}]-(E[\tilde{\gamma}])^{2}} .
\end{gathered}
$$

Proof. From Eq. (11), the first order conditions can be obtained as

$$
\begin{aligned}
& \frac{\partial}{\partial w} E\left[\Pi_{M}\right]=-2 E[\tilde{\beta}] w+E[\tilde{\gamma}] \theta+E[\tilde{D}]+E[\tilde{\beta} \tilde{c}]-E[\tilde{\beta}] m . \\
& \frac{\partial}{\partial \theta} E\left[\Pi_{M}\right]=-2 E[\tilde{I}] \theta+E[\tilde{\gamma}] w-\frac{1}{2} \int_{0}^{1}\left(\tilde{\gamma}_{\alpha}^{L} \tilde{c}_{\alpha}^{R}+\tilde{\gamma}_{\alpha}^{R} \tilde{c}_{\alpha}^{L}\right) \mathrm{d} \alpha .
\end{aligned}
$$

Therefore the Hessian matrix of $E\left[\Pi_{M}\right]$ is

$$
\mathrm{H}=\left[\begin{array}{cc}
-2 E[\tilde{\beta}] & E[\tilde{\gamma}] \\
E[\tilde{\gamma}] & -2 E[\tilde{I}]
\end{array}\right] .
$$

Since $E[\tilde{\beta}]>0, E[\tilde{I}]>0$ and $(E[\tilde{\gamma}])^{2}<4 E[\tilde{\beta}] E[\tilde{I}]$, thus $\mathrm{H}$ is a definite negative matrix and $E\left[\Pi_{M}\right]$ is jointly concave in $w$ and $\theta$.

Let the first order conditions be zero, we can have Eqs.(26) and (27).

Theorem 6 is proved.

Here, $w(m)$ and $\theta(m)$ can be regarded as the manufacture's response functions to the margin profit $m$. The retailer sets his optimal decisions. So, the following Proposition can be derived.

Theorem 7. Let $E\left[\Pi_{R}(m)\right]$ be the retailer's fuzzy expected profit. If $(E[\tilde{\gamma}])^{2}<4 E[\tilde{\beta}] E[\tilde{I}], \operatorname{Pos}\left\{w^{* *}-\tilde{c}<0\right\}=0$ and $\operatorname{Pos}\left\{\tilde{D}-\tilde{\beta}\left(m^{* *}+w^{* *}\right)+\tilde{\gamma} \theta^{* *}<0\right\}=0$, then the optimal equilibrium decisions are

$$
\begin{aligned}
m^{* *}= & \frac{E[\tilde{\gamma}]\left(2 E[\tilde{\beta} \tilde{c}] E[\tilde{\gamma}]-E[\tilde{\beta}] \int_{0}^{1}\left(\tilde{\gamma}_{\alpha}^{L} \tilde{c}_{\alpha}^{R}+\tilde{\gamma}_{\alpha}^{R} \tilde{c}_{\alpha}^{L}\right) \mathrm{d} \alpha\right)}{8(E[\tilde{\beta}])^{2} E[\tilde{I}]} \\
& +\frac{(E[\tilde{D}]-E[\tilde{\beta} \tilde{c}])}{2 E[\tilde{\beta}]} .
\end{aligned}
$$




$$
\begin{aligned}
& w^{* *}= \frac{E[\tilde{I}](E[\tilde{D}]+3 E[\tilde{\beta} \tilde{c}])}{4 E[\tilde{\beta}] E[\tilde{I}]-(E[\tilde{\gamma}])^{2}} \\
&-\frac{E[\tilde{\gamma}]\left(2 E[\tilde{\beta} \tilde{c}] E[\tilde{\gamma}]+E[\tilde{\beta}] \int_{0}^{1}\left(\tilde{\gamma}_{\alpha}^{L} \tilde{c}_{\alpha}^{R}+\tilde{\gamma}_{\alpha}^{R} \tilde{c}_{\alpha}^{L}\right) \mathrm{d} \alpha\right)}{4 E[\tilde{\beta}]\left(4 E[\tilde{\beta}] E[\tilde{I}]-(E[\tilde{\gamma}])^{2}\right)} . \\
& \theta^{* *}=\frac{E[\tilde{\gamma}](E[\tilde{D}]+3 E[\tilde{\beta} \tilde{c}])}{2\left(4 E[\tilde{\beta}] E[\tilde{I}]-(E[\tilde{\gamma}])^{2}\right)} \\
&-\frac{\left(8 E[\tilde{\beta}] E[\tilde{I}]-(E[\tilde{\gamma}])^{2}\right) \int_{0}^{1}\left(\tilde{\gamma}_{\alpha}^{L} \tilde{c}_{\alpha}^{R}+\tilde{\gamma}_{\alpha}^{R} \tilde{c}_{\alpha}^{L}\right) \mathrm{d} \alpha}{8 E[\tilde{I}]\left(4 E[\tilde{\beta}] E[\tilde{I}]-(E[\tilde{\gamma}])^{2}\right)} \\
&-\frac{E[\tilde{\beta} \tilde{c}](E[\tilde{\gamma}])^{3}}{4 E[\tilde{\beta}] E[\tilde{I}]\left(4 E[\tilde{\beta}] E[\tilde{I}]-(E[\tilde{\gamma}])^{2}\right)} .
\end{aligned}
$$

Proof. Substituting Eqs. (26) and (27) into Eq. (7), the first order condition is

$$
\begin{gathered}
\frac{\mathrm{d}}{\mathrm{d} m} E\left[\Pi_{R}(m)\right]=-\frac{4(E[\tilde{\beta}])^{2} E[\tilde{I}]}{4 E[\tilde{\beta}] E[\tilde{\gamma}]-(E[\tilde{I}])^{2}} m \\
+\frac{2 E[\tilde{\beta}] E[\tilde{I}](E[\tilde{D}]-E[\tilde{\beta} \tilde{c}])}{4 E[\tilde{\beta}] E[\tilde{\gamma}]-(E[\tilde{I}])^{2}} \\
+\frac{E[\tilde{\gamma}]\left(2 E[\tilde{\beta} \tilde{c}] E[\tilde{\gamma}]-E[\tilde{\beta}] \int_{0}^{1}\left(\tilde{\gamma}_{\alpha}^{L} \tilde{c}_{\alpha}^{R}+\tilde{\gamma}_{\alpha}^{R} \tilde{c}_{\alpha}^{L}\right) \mathrm{d} \alpha\right)}{2\left(4 E[\tilde{\beta}] E[\tilde{\gamma}]-(E[\tilde{I}])^{2}\right)} .
\end{gathered}
$$

The second order condition is

$$
\frac{\mathrm{d}^{2}}{\mathrm{~d} m^{2}} E\left[\Pi_{R}(m)\right]=-\frac{4(E[\tilde{\beta}])^{2} E[\tilde{I}]}{4 E[\tilde{\beta}] E[\tilde{I}]-(E[\tilde{\gamma}])^{2}} .
$$

Since $E[\tilde{\beta}]>0, E[\tilde{\gamma}]>0, E[\tilde{I}]>0$ and $(E[\tilde{\gamma}])^{2}<4 E[\tilde{\beta}] E[\tilde{I}]$ , then the second order condition is negative definite. Thus, $E\left[\Pi_{R}(m)\right]$ is concave in $m$.

Let the first order conditions be zero, we can have Eq.(28).

Substituting $m^{* *}$ in Eq. (28) into Eqs. (26) and (27), we can get Eqs. (29) and (30).

Theorem 7 is proved.

In the RS game, the maximax chance-constrained programming model of GSC can be formulated as

$$
\begin{aligned}
& \max _{\substack{m \\
\text { s.t. }}}\left(\Pi_{R}(m)\right)_{\alpha}^{R}=\left(m\left(\tilde{D}-\tilde{\beta}\left(m+w^{* *}\right)+\tilde{\gamma} \theta^{* *}\right)\right)_{\alpha}^{R} \\
& \text { where } w^{* * *} \text { and } \theta^{* * *} \text { derive from problem: } \\
& \left\{\begin{array}{l}
\max _{w, \theta}\left(\Pi_{M}(w, \theta)\right)_{\alpha}^{R}=\left((w-\tilde{c})(\tilde{D}-\tilde{\beta}(m+w)+\tilde{\gamma} \theta)-\tilde{I} \theta^{2}\right)_{\alpha}^{R} . \\
\text { s.t. } \\
\operatorname{Pos}\left\{w^{* *}-\tilde{c}<0\right\}=0 \\
\operatorname{Pos}\left\{\tilde{D}-\tilde{\beta}\left(m+w^{* *}\right)+\tilde{\gamma} \theta^{* *}<0\right\}=0
\end{array}\right.
\end{aligned}
$$

Theorem 8. Let $\left(\Pi_{M}(w, \theta)\right)_{\alpha}^{R}$ be the manufacturer's $\alpha$ optimistic value of profit. If $\left(\tilde{\gamma}_{\alpha}^{R}\right)^{2}<4 \tilde{\beta}_{\alpha}^{L} \tilde{I}_{\alpha}^{L}$, $\operatorname{Pos}\left\{w^{* *}-\tilde{c}<0\right\}=0$ and $\operatorname{Pos}\left\{\tilde{D}-\tilde{\beta}\left(m+w^{* *}\right)+\tilde{\gamma} \theta^{* *}<0\right\}=0$, then the optimal reaction functions for manufacturer are

$$
\begin{aligned}
w^{* *}(m) & =\frac{2 \tilde{I}_{\alpha}^{L}\left(\tilde{D}_{\alpha}^{R}+\tilde{\beta}_{\alpha}^{L} \tilde{c}_{\alpha}^{L}\right)-2 \tilde{\beta}_{\alpha}^{L} \tilde{I}_{\alpha}^{L} m-\left(\tilde{\gamma}_{\alpha}^{R}\right)^{2} \tilde{c}_{\alpha}^{L}}{4 \tilde{\beta}_{\alpha}^{L} \tilde{I}_{\alpha}^{L}-\left(\tilde{\gamma}_{\alpha}^{R}\right)^{2}} . \\
\theta^{* *}(m) & =\frac{\tilde{\gamma}_{\alpha}^{R}\left(\tilde{D}_{\alpha}^{R}-\tilde{\beta}_{\alpha}^{L} \tilde{c}_{\alpha}^{L}-\tilde{\beta}_{\alpha}^{L} m\right)}{4 \tilde{\beta}_{\alpha}^{L} \tilde{I}_{\alpha}^{L}-\left(\tilde{\gamma}_{\alpha}^{R}\right)^{2}} .
\end{aligned}
$$

Proof. From Eq. (19), the first order conditions can be obtained as

$$
\begin{aligned}
& \frac{\partial}{\partial w}\left(\Pi_{M}\left(w, \theta, m^{*}\right)\right)_{\alpha}^{R}=-2 \tilde{\beta}_{\alpha}^{L} w+\tilde{\gamma}_{\alpha}^{R} \theta-\tilde{\beta}_{\alpha}^{L} m+\tilde{D}_{\alpha}^{R}+\tilde{\beta}_{\alpha}^{L} \tilde{c}_{\alpha}^{L} . \\
& \frac{\partial}{\partial \theta}\left(\Pi_{M}\left(w, \theta, m^{*}\right)\right)_{\alpha}^{R}=-2 \tilde{I}_{\alpha}^{L} \theta+\tilde{\gamma}_{\alpha}^{R} w-\tilde{\gamma}_{\alpha}^{R} \tilde{c}_{\alpha}^{L} .
\end{aligned}
$$

Therefore, the Hessian matrix of $\left(\Pi_{M}\left(w, \theta, m^{*}\right)\right)_{\alpha}^{R}$ is

$$
\mathrm{H}=\left[\begin{array}{cc}
-2 \tilde{\beta}_{\alpha}^{L} & \tilde{\gamma}_{\alpha}^{R} \\
\tilde{\gamma}_{\alpha}^{R} & -2 \tilde{I}_{\alpha}^{L}
\end{array}\right] .
$$

Since $\tilde{\beta}, \tilde{I}$ are positive fuzzy variables and $\left(\tilde{\gamma}_{\alpha}^{R}\right)^{2}<4 \tilde{\beta}_{\alpha}^{L} \tilde{I}_{\alpha}^{L}$, thus, for any $\alpha \in(0,1], \mathrm{H}$ is a definite negative matrix and $\left(\Pi_{M}(w, \theta)\right)_{\alpha}^{R}$ is jointly concave in $w$ and $\theta$.

Let the first order conditions be zero, the results (32) and (33) can be derived.

Theorem 8 is proved.

Theorem 9. Let $\left(\Pi_{R}(m)\right)_{\alpha}^{R}$ be the retailer's $\alpha$-optimistic value of profit. If $\left(\tilde{\gamma}_{\alpha}^{R}\right)^{2}<4 \tilde{\beta}_{\alpha}^{L} \tilde{I}_{\alpha}^{L}, \operatorname{Pos}\left\{w^{* *}-\tilde{c}<0\right\}=0$ and $\operatorname{Pos}\left\{\tilde{D}-\tilde{\beta}\left(m^{* *}+w^{* *}\right)+\tilde{\gamma} \theta^{* * *}<0\right\}=0$, then the optimal equilibrium decisions are

$$
m^{* *}=\frac{\tilde{D}_{\alpha}^{R}-\tilde{\beta}_{\alpha}^{L} \tilde{c}_{\alpha}^{L}}{2 \tilde{\beta}_{\alpha}^{L}} .
$$




$$
\begin{aligned}
& w^{* *}=\frac{\tilde{I}_{\alpha}^{L}\left(\tilde{D}_{\alpha}^{R}+3 \tilde{\beta}_{\alpha}^{L} \tilde{c}_{\alpha}^{L}\right)-\left(\tilde{\gamma}_{\alpha}^{R}\right)^{2} \tilde{c}_{\alpha}^{L}}{4 \tilde{\beta}_{\alpha}^{L} \tilde{I}_{\alpha}^{L}-\left(\tilde{\gamma}_{\alpha}^{R}\right)^{2}} . \\
& \theta^{* *}=\frac{\tilde{\gamma}_{\alpha}^{R}\left(\tilde{D}_{\alpha}^{R}-\tilde{\beta}_{\alpha}^{L} \tilde{c}_{\alpha}^{L}\right)}{2\left(4 \tilde{\beta}_{\alpha}^{L} \tilde{I}_{\alpha}^{L}-\left(\tilde{\gamma}_{\alpha}^{R}\right)^{2}\right)} .
\end{aligned}
$$

Proof. Substituting $w^{* *}(m)$ and $\theta^{* *}(m)$ in Eqs. (32) and (33) into Eq. (15), the first order condition is

$$
\frac{\mathrm{d}}{\mathrm{d} m}\left(\Pi_{R}(m)\right)_{\alpha}^{R}=-\frac{4\left(\tilde{\beta}_{\alpha}^{L}\right)^{2} \tilde{I}_{\alpha}^{L}}{4 \tilde{\beta}_{\alpha}^{L} \tilde{I}_{\alpha}^{L}-\tilde{\gamma}_{\alpha}^{R}} m+\frac{2 \tilde{\beta}_{\alpha}^{L} \tilde{I}_{\alpha}^{L}\left(\tilde{D}_{\alpha}^{R}-\tilde{\beta}_{\alpha}^{L} \tilde{c}_{\alpha}^{L}\right)}{4 \tilde{\beta}_{\alpha}^{L} \tilde{I}_{\alpha}^{L}-\tilde{\gamma}_{\alpha}^{R}} .
$$

The second order condition is

$$
\frac{\mathrm{d}^{2}}{\mathrm{~d} m^{2}}\left(\Pi_{R}(m)\right)_{\alpha}^{R}=-\frac{4\left(\tilde{\beta}_{\alpha}^{L}\right)^{2} \tilde{I}_{\alpha}^{L}}{4 \tilde{\beta}_{\alpha}^{L} \tilde{I}_{\alpha}^{L}-\tilde{\gamma}_{\alpha}^{R}} .
$$

Since $\tilde{\beta}, \tilde{I}$ and $\tilde{\gamma}$ are positive fuzzy variables, and $\left(\tilde{\gamma}_{\alpha}^{R}\right)^{2}<4 \tilde{\beta}_{\alpha}^{L} \tilde{I}_{\alpha}^{L}$, then the second order condition is negative definite. Thus, for any $\alpha \in(0,1],\left(\Pi_{R}(m)\right)_{\alpha}^{R}$ is concave in $m$.

Let $\frac{\mathrm{d}}{\mathrm{d} m}\left(\Pi_{R}(m)\right)_{\alpha}^{R}=0$, we can get the retailer's optimal margin profit as in Eq. (34).

Substituting $m^{* *}$ in Eq. (34) into Eqs. (32) and (33), we can get Eqs. (35) and (36).

Theorem 9 is proved.

The optimal optimistic profits of the manufacturer and the retailer can be easily obtained as follows

$$
\begin{gathered}
\left(\Pi_{M}\right)_{\alpha}^{R}=\frac{\tilde{I}_{\alpha}^{L}\left(\tilde{D}_{\alpha}^{R}-\tilde{\beta}_{\alpha}^{L} \tilde{c}_{\alpha}^{L}\right)^{2}}{4\left(4 \tilde{\beta}_{\alpha}^{L} \tilde{I}_{\alpha}^{L}-\left(\tilde{\gamma}_{\alpha}^{R}\right)^{2}\right)} . \\
\left(\Pi_{R}\right)_{\alpha}^{R}=\frac{\tilde{I}_{\alpha}^{L}\left(\tilde{D}_{\alpha}^{R}-\tilde{\beta}_{\alpha}^{L} \tilde{c}_{\alpha}^{L}\right)^{2}}{2\left(4 \tilde{\beta}_{\alpha}^{L} \tilde{I}_{\alpha}^{L}-\left(\tilde{\gamma}_{\alpha}^{R}\right)^{2}\right)} .
\end{gathered}
$$

In the RS game, the minimax chance-constrained programming model of GSC can be formulate as follows

$$
\begin{aligned}
& \max _{\substack{m \\
\text { s.t. }}}\left(\Pi_{R}(m)\right)_{\alpha}^{L}=\left(m\left(\tilde{D}-\tilde{\beta}\left(m+w^{* *}\right)+\tilde{\gamma} \theta^{* *}\right)\right)_{\alpha}^{L} \\
& \text { where } w^{* *} \text { and } \theta^{* *} \text { derive from problem: } \\
& \left\{\begin{array}{l}
\max _{w, \theta}\left(\Pi_{M}(w, \theta)\right)_{\alpha}^{L}=\left((w-\tilde{c})(\tilde{D}-\tilde{\beta}(m+w)+\tilde{\gamma} \theta)-\tilde{I} \theta^{2}\right)_{\alpha}^{L} \\
\text { s.t.t. } \\
\operatorname{Pos}\left\{w^{* *}-\tilde{c}<0\right\}=0 \\
\operatorname{Pos}\left\{\tilde{D}-\tilde{\beta}\left(m+w^{* *}\right)+\tilde{\gamma} \theta^{* *}<0\right\}=0
\end{array}\right.
\end{aligned}
$$

Theorem 10. Let $\left(\Pi_{R}(m)\right)_{\alpha}^{L}$ and $\left(\Pi_{M}(w, \theta)\right)_{\alpha}^{L}$ be the $\alpha$ pessimistic value of profit for the retailer and the manufacturer. If $\left(\tilde{\gamma}_{\alpha}^{L}\right)^{2}<4 \tilde{\beta}_{\alpha}^{R} \tilde{I}_{\alpha}^{R}, \quad \operatorname{Pos}\left\{w^{* *}-\tilde{c}<0\right\}=0$ and $\operatorname{Pos}\left\{\tilde{D}-\tilde{\beta}\left(m^{* *}+w^{* *}\right)+\tilde{\gamma} \theta^{* *}<0\right\}=0$, then the optimal equilibrium decisions are

$$
\begin{aligned}
m^{* *} & =\frac{\tilde{D}_{\alpha}^{L}-\tilde{\beta}_{\alpha}^{R} \tilde{c}_{\alpha}^{R}}{2 \tilde{\beta}_{\alpha}^{R}} . \\
w^{* *} & =\frac{\tilde{I}_{\alpha}^{R}\left(\tilde{D}_{\alpha}^{L}+3 \tilde{\beta}_{\alpha}^{R} \tilde{c}_{\alpha}^{R}\right)-\left(\tilde{\gamma}_{\alpha}^{L}\right)^{2} \tilde{c}_{\alpha}^{R}}{4 \tilde{\beta}_{\alpha}^{R} \tilde{I}_{\alpha}^{R}-\left(\tilde{\gamma}_{\alpha}^{L}\right)^{2}} . \\
\theta^{* * *} & =\frac{\tilde{\gamma}_{\alpha}^{L}\left(\tilde{D}_{\alpha}^{L}-\tilde{\beta}_{\alpha}^{R} \tilde{c}_{\alpha}^{R}\right)}{2\left(4 \tilde{\beta}_{\alpha}^{R} \tilde{I}_{\alpha}^{R}-\left(\tilde{\gamma}_{\alpha}^{L}\right)^{2}\right)} .
\end{aligned}
$$

Proof. Similar to Theorems 8 and 9.

The optimal pessimistic profits for the supply chain members are given by

$$
\begin{gathered}
\left(\Pi_{M}\right)_{\alpha}^{L}=\frac{\tilde{I}_{\alpha}^{R}\left(\tilde{D}_{\alpha}^{L}-\tilde{\beta}_{\alpha}^{R} \tilde{c}_{\alpha}^{R}\right)^{2}}{4\left(4 \tilde{\beta}_{\alpha}^{R} \tilde{I}_{\alpha}^{R}-\left(\tilde{\gamma}_{\alpha}^{L}\right)^{2}\right)} . \\
\left(\Pi_{R}\right)_{\alpha}^{L}=\frac{\tilde{I}_{\alpha}^{R}\left(\tilde{D}_{\alpha}^{L}-\tilde{\beta}_{\alpha}^{R} \tilde{c}_{\alpha}^{R}\right)^{2}}{2\left(4 \tilde{\beta}_{\alpha}^{R} \tilde{I}_{\alpha}^{R}-\left(\tilde{\gamma}_{\alpha}^{L}\right)^{2}\right)} .
\end{gathered}
$$

Remark 2. If $\alpha=1$, then the parameters $\tilde{c}, \tilde{D}, \tilde{\beta}$, $\tilde{\gamma}$ and $\tilde{I}$ reduce to crisp numbers, then the optimal solutions in Theorems 9 and 10 are

$$
\begin{aligned}
m^{* *} & =\frac{D-\beta c}{2 \beta} . \\
w^{* *} & =\frac{I(D+3 \beta c)-\gamma^{2} c}{4 \beta I-\gamma^{2}} . \\
\theta^{* *} & =\frac{\gamma(D-\beta c)}{2\left(4 \beta I-\gamma^{2}\right)} .
\end{aligned}
$$

\subsection{Vertical Nash (VN) game}

In the VN game, the supply chain participants have an equal bargaining power. Thus, in this condition, the manufacturer makes his wholesale price $w$ and level of greening innovation $\theta$, and the retailer makes his margin profit $m$ simultaneously and independently. The assumption VN game means a Nash equilibrium among the manufacturer and the retailer, hence, in this case, we can formulate the fuzzy optimal model as follows 


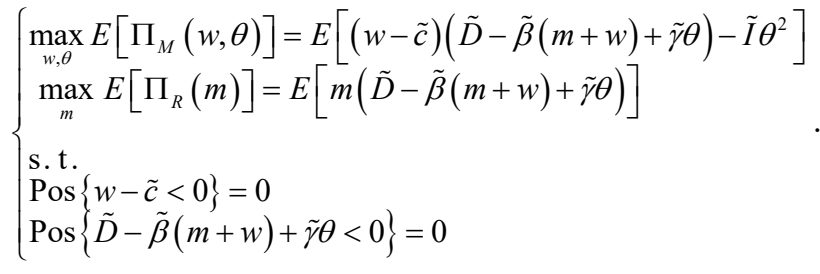

Theorem 11. Let $E\left[\Pi_{M}(w, \theta)\right]$ and $E\left[\Pi_{R}(m)\right]$ be the manufacturer's and retailer's fuzzy expected profits. If $(E[\tilde{\gamma}])^{2}<4 E[\tilde{\beta}] E[\tilde{I}], \operatorname{Pos}\left\{\tilde{D}-\tilde{\beta}\left(m^{* * *}+w^{* * *}\right)+\tilde{\gamma} \theta^{* * *}<0\right\}=0$ and $\operatorname{Pos}\left\{w^{* * *}-\tilde{c}<0\right\}=0$, then the optimal equilibrium decisions are

$$
\begin{aligned}
m^{* * * *}= & \frac{E[\tilde{\gamma}]\left(2 E[\tilde{\beta} \tilde{c}] E[\tilde{\gamma}]-E[\tilde{\beta}] \int_{0}^{1}\left(\tilde{\gamma}_{\alpha}^{L} \tilde{c}_{\alpha}^{R}+\tilde{\gamma}_{\alpha}^{R} \tilde{c}_{\alpha}^{L}\right) \mathrm{d} \alpha\right)}{2 E[\tilde{\beta}]\left(6 E[\tilde{\beta}] E[\tilde{I}]-(E[\gamma])^{2}\right)} \\
& +\frac{2 E[\tilde{I}](E[\tilde{D}]-E[\tilde{\beta} \tilde{c}])}{6 E[\tilde{\beta}] E[\tilde{I}]-(E[\gamma])^{2}} . \\
w^{* * *}= & \frac{4 E[\tilde{I}](E[\tilde{D}]+2 E[\tilde{\beta} \tilde{c}])-E[\tilde{\gamma}] \int_{0}^{1}\left(\tilde{\gamma}_{\alpha}^{L} \tilde{c}_{\alpha}^{R}+\tilde{\gamma}_{\alpha}^{R} \tilde{c}_{\alpha}^{L}\right) \mathrm{d} \alpha}{2\left(6 E[\tilde{\beta}] E[\tilde{I}]-(E[\gamma])^{2}\right)} .
\end{aligned}
$$

$$
\theta^{* * *}=\frac{2 E[\tilde{\gamma}](E[\tilde{D}]+2 E[\tilde{\beta} \tilde{c}])-3 E[\tilde{\beta}] \int_{0}^{1}\left(\tilde{\gamma}_{\alpha}^{L} \tilde{c}_{\alpha}^{R}+\tilde{\gamma}_{\alpha}^{R} \tilde{c}_{\alpha}^{L}\right) \mathrm{d} \alpha}{2\left(6 E[\tilde{\beta}] E[\tilde{I}]-(E[\gamma])^{2}\right)} .
$$

Proof. From Eq. (7), the first order condition is

$$
\frac{\mathrm{d}}{\mathrm{d} m} E\left[\Pi_{R}(m)\right]=-2 E[\tilde{\beta}] m+E[\tilde{D}]-E[\tilde{\beta}] w+E[\tilde{\gamma}] \theta .
$$

The second order condition is

$$
\frac{\mathrm{d}^{2}}{\mathrm{~d} m^{2}} E\left[\Pi_{R}(m)\right]=-2 E[\tilde{\beta}]<0 .
$$

Thus, the retailer's fuzzy expected profit $E\left[\Pi_{R}(m)\right]$ is strictly concave in $m$.

From Eq. (11), the first order conditions are

$$
\begin{aligned}
& \frac{\partial}{\partial w} E\left[\Pi_{M}\right]=-2 E[\tilde{\beta}] w+E[\tilde{\gamma}] \theta+E[\tilde{D}]+E[\tilde{\beta} \tilde{c}]-E[\tilde{\beta}] m . \\
& \frac{\partial}{\partial \theta} E\left[\Pi_{M}\right]=-2 E[\tilde{I}] \theta+E[\tilde{\gamma}] w-\frac{1}{2} \int_{0}^{1}\left(\tilde{\gamma}_{\alpha}^{L} \tilde{c}_{\alpha}^{R}+\tilde{\gamma}_{\alpha}^{R} \tilde{c}_{\alpha}^{L}\right) \mathrm{d} \alpha .
\end{aligned}
$$

Therefore, the Hessian matrix of $E\left[\Pi_{M}\right]$ is

$$
\mathrm{H}=\left[\begin{array}{cc}
-2 E[\tilde{\beta}] & E[\tilde{\gamma}] \\
E[\tilde{\gamma}] & -2 E[\tilde{I}]
\end{array}\right]
$$

Since $E[\tilde{\beta}]>0, E[\tilde{I}]>0$ and $(E[\tilde{\gamma}])^{2}<4 E[\tilde{\beta}] E[\tilde{I}]$, thus $\mathrm{H}$ is a definite negative matrix, and $E\left[\Pi_{M}\right]$ is jointly concave in $w$ and $\theta$.

We can have Eqs. (42), (43) and (44) by setting the first order conditions above be zero.

Theorem 11 is proved.

In the VN game, we can also formulate the minimax chance-constrained programming model of GSC as

$$
\left\{\begin{array}{l}
\max _{w, \theta}\left(\Pi_{M}(w, \theta)\right)_{\alpha}^{R}=\left((w-\tilde{c})(\tilde{D}-\tilde{\beta}(m+w)+\tilde{\gamma} \theta)-\tilde{I} \theta^{2}\right)_{\alpha}^{R} \\
\max _{m}\left(\Pi_{R}(m)\right)_{\alpha}^{R}=(m(\tilde{D}-\tilde{\beta}(m+w)+\tilde{\gamma} \theta))_{\alpha}^{R} \\
\text { s.t. } \\
\operatorname{Pos}\{w-\tilde{c}<0\}=0 \\
\operatorname{Pos}\{\tilde{D}-\tilde{\beta}(m+w)+\tilde{\gamma} \theta<0\}=0
\end{array}\right.
$$

Theorem 12. Let $\left(\Pi_{M}(w, \theta)\right)_{\alpha}^{R}$ and $\left(\Pi_{R}(m)\right)_{\alpha}^{R}$ be the manufacturer's and the retailer's $\alpha$-optimistic value of profits. If $\left(\tilde{\gamma}_{\alpha}^{R}\right)^{2}<4 \tilde{\beta}_{\alpha}^{L} \tilde{I}_{\alpha}^{L}, \operatorname{Pos}\left\{w^{* * *}-\tilde{c}<0\right\}=0$ and $\operatorname{Pos}\left\{\tilde{D}-\tilde{\beta}\left(m^{* * *}+w^{* * *}\right)+\tilde{\gamma} \theta^{* * *}<0\right\}=0$, then the optimal equilibrium decisions are

$$
\begin{aligned}
m^{* * *} & =\frac{2 \tilde{I}_{\alpha}^{L}\left(\tilde{D}_{\alpha}^{R}-\tilde{\beta}_{\alpha}^{L} \tilde{c}_{\alpha}^{L}\right)}{6 \tilde{\beta}_{\alpha}^{L} \tilde{I}_{\alpha}^{L}-\left(\tilde{\gamma}_{\alpha}^{R}\right)^{2}} . \\
w^{* * *} & =\frac{2 \tilde{I}_{\alpha}^{L}\left(\tilde{D}_{\alpha}^{R}+2 \tilde{\beta}_{\alpha}^{L} \tilde{c}_{\alpha}^{L}\right)-\left(\tilde{\gamma}_{\alpha}^{R}\right)^{2} \tilde{c}_{\alpha}^{L}}{6 \tilde{\beta}_{\alpha}^{L} \tilde{I}_{\alpha}^{L}-\left(\tilde{\gamma}_{\alpha}^{R}\right)^{2}} . \\
\theta^{* * *} & =\frac{\tilde{\gamma}_{\alpha}^{R}\left(\tilde{D}_{\alpha}^{R}-\tilde{\beta}_{\alpha}^{L} \tilde{c}_{\alpha}^{L}\right)}{6 \tilde{\beta}_{\alpha}^{L} \tilde{I}_{\alpha}^{L}-\left(\tilde{\gamma}_{\alpha}^{R}\right)^{2}} .
\end{aligned}
$$

Proof. From Eq. (15), the first order condition is

$$
\frac{\mathrm{d}}{\mathrm{d} m}\left(\Pi_{R}(m)\right)_{\alpha}^{R}=-2 \tilde{\beta}_{\alpha}^{L} m+\tilde{D}_{\alpha}^{R}-\tilde{\beta}_{\alpha}^{L} w+\tilde{\gamma}_{\alpha}^{R} \theta .
$$

The second order condition is

$$
\frac{\mathrm{d}^{2}}{\mathrm{~d} m^{2}}\left(\Pi_{R}(m)\right)_{\alpha}^{R}=-2 \tilde{\beta}_{\alpha}^{L}<0 .
$$

Thus, for any $\alpha \in(0,1],\left(\Pi_{R}(m)\right)_{\alpha}^{R}$ is concave in $m$. From Eq. (19), the first order conditions are 


$$
\begin{aligned}
& \frac{\partial}{\partial w}\left(\Pi_{M}\left(w, \theta, m^{*}\right)\right)_{\alpha}^{R}=-2 \tilde{\beta}_{\alpha}^{L} w+\tilde{\gamma}_{\alpha}^{R} \theta-\tilde{\beta}_{\alpha}^{L} m+\tilde{D}_{\alpha}^{R}+\tilde{\beta}_{\alpha}^{L} \tilde{c}_{\alpha}^{L} . \\
& \frac{\partial}{\partial \theta}\left(\Pi_{M}\left(w, \theta, m^{*}\right)\right)_{\alpha}^{R}=-2 \tilde{I}_{\alpha}^{L} \theta+\tilde{\gamma}_{\alpha}^{R} w-\tilde{\gamma}_{\alpha}^{R} \tilde{c}_{\alpha}^{L} .
\end{aligned}
$$

Therefore, the Hessian matrix of $\left(\Pi_{M}\left(w, \theta, m^{*}\right)\right)_{\alpha}^{R}$ is

$$
\mathrm{H}=\left[\begin{array}{cc}
-2 \tilde{\beta}_{\alpha}^{L} & \tilde{\gamma}_{\alpha}^{R} \\
\tilde{\gamma}_{\alpha}^{R} & -2 \tilde{I}_{\alpha}^{L}
\end{array}\right] .
$$

Since $\tilde{\beta}, \tilde{I}$ are positive fuzzy variables and $\left(\tilde{\gamma}_{\alpha}^{R}\right)^{2}<4 \tilde{\beta}_{\alpha}^{L} \tilde{I}_{\alpha}^{L}$, then the Hessian matrix $\mathrm{H}$ is negative definite. Thus, for any $\alpha \in(0,1],\left(\Pi_{M}(w, \theta)\right)_{\alpha}^{R}$ is jointly concave in $w$ and $\theta$.

We can have Eqs. (46), (47) and (48) by setting the first order conditions above be zero.

Theorem 12 is proved.

The optimal optimistic profits of the manufacturer and the retailer can be easily obtained as follows

$$
\begin{aligned}
&\left(\Pi_{M}\right)_{\alpha}^{R}= \frac{\tilde{I}_{\alpha}^{L}\left(4 \tilde{\beta}_{\alpha}^{L} \tilde{I}_{\alpha}^{L}-\left(\tilde{\gamma}_{\alpha}^{R}\right)^{2}\right)\left(\tilde{D}_{\alpha}^{R}-\tilde{\beta}_{\alpha}^{L} \tilde{c}_{\alpha}^{L}\right)^{2}}{\left(6 \tilde{\beta}_{\alpha}^{L} \tilde{I}_{\alpha}^{L}-\left(\tilde{\gamma}_{\alpha}^{R}\right)^{2}\right)^{2}} . \\
&\left(\Pi_{R}\right)_{\alpha}^{R}=\frac{4 \tilde{\beta}_{\alpha}^{L}\left(\tilde{I}_{\alpha}^{L}\right)^{2}\left(\tilde{D}_{\alpha}^{R}-\tilde{\beta}_{\alpha}^{L} \tilde{c}_{\alpha}^{L}\right)^{2}}{\left(6 \tilde{\beta}_{\alpha}^{L} \tilde{I}_{\alpha}^{L}-\left(\tilde{\gamma}_{\alpha}^{R}\right)^{2}\right)^{2}} .
\end{aligned}
$$

In the $\mathrm{VN}$ game, the minimax chance-constrained programming model of GSC can be formulated as follows

$$
\left\{\begin{array}{l}
\max _{w, \theta}\left(\Pi_{M}(w, \theta)\right)_{\alpha}^{L}=\left((w-\tilde{c})(\tilde{D}-\tilde{\beta}(m+w)+\tilde{\gamma} \theta)-\tilde{I} \theta^{2}\right)_{\alpha}^{L} \\
\max _{m}\left(\Pi_{R}(m)\right)_{\alpha}^{L}=(m(\tilde{D}-\tilde{\beta}(m+w)+\tilde{\gamma} \theta))_{\alpha}^{L} \\
\text { s.t. } \\
\text { Pos }\{w-\tilde{c}<0\}=0 \\
\operatorname{Pos}\{\tilde{D}-\tilde{\beta}(m+w)+\tilde{\gamma} \theta<0\}=0
\end{array}\right.
$$

Theorem 13. Let $\left(\Pi_{M}(w, \theta)\right)_{\alpha}^{L}$ and $\left(\Pi_{R}(m)\right)_{\alpha}^{L}$ be the manufacturer's and retailer's $\alpha$-pessimistic value of profits. If $\left(\tilde{\gamma}_{\alpha}^{L}\right)^{2}<4 \tilde{\beta}_{\alpha}^{R} \tilde{I}_{\alpha}^{R}, \operatorname{Pos}\left\{w^{* * *}-\tilde{c}<0\right\}=0$ and $\operatorname{Pos}\left\{\tilde{D}-\tilde{\beta}\left(m^{* * *}+w^{* * *}\right)+\tilde{\gamma} \theta^{* * * *}<0\right\}=0$, then the optimal equilibrium decisions are

$$
\begin{aligned}
m^{* * * *} & =\frac{2 \tilde{I}_{\alpha}^{R}\left(\tilde{D}_{\alpha}^{L}-\tilde{\beta}_{\alpha}^{R} \tilde{c}_{\alpha}^{R}\right)}{6 \tilde{\beta}_{\alpha}^{R} \tilde{I}_{\alpha}^{R}-\left(\tilde{\gamma}_{\alpha}^{L}\right)^{2}} . \\
w^{* * * *} & =\frac{2 \tilde{I}_{\alpha}^{R}\left(\tilde{D}_{\alpha}^{L}+2 \tilde{\beta}_{\alpha}^{R} \tilde{c}_{\alpha}^{R}\right)-\left(\tilde{\gamma}_{\alpha}^{L}\right)^{2} \tilde{c}_{\alpha}^{R}}{6 \tilde{\beta}_{\alpha}^{R} \tilde{I}_{\alpha}^{R}-\left(\tilde{\gamma}_{\alpha}^{L}\right)^{2}} . \\
\theta^{* * * *} & =\frac{\tilde{\gamma}_{\alpha}^{L}\left(\tilde{D}_{\alpha}^{L}-\tilde{\beta}_{\alpha}^{R} \tilde{c}_{\alpha}^{R}\right)}{6 \tilde{\beta}_{\alpha}^{R} \tilde{I}_{\alpha}^{R}-\left(\tilde{\gamma}_{\alpha}^{L}\right)^{2}} .
\end{aligned}
$$

Proof. Similar to Theorem 12.

The optimal pessimistic profits of the manufacturer and the retailer can be obtained as follows

$$
\begin{aligned}
\left(\Pi_{M}\right)_{\alpha}^{L} & =\frac{\tilde{I}_{\alpha}^{R}\left(4 \tilde{\beta}_{\alpha}^{R} \tilde{I}_{\alpha}^{R}-\left(\tilde{\gamma}_{\alpha}^{L}\right)^{2}\right)\left(\tilde{D}_{\alpha}^{L}-\tilde{\beta}_{\alpha}^{R} \tilde{c}_{\alpha}^{R}\right)^{2}}{\left(6 \tilde{\beta}_{\alpha}^{R} \tilde{I}_{\alpha}^{R}-\left(\tilde{\gamma}_{\alpha}^{L}\right)^{2}\right)^{2}} . \\
\left(\Pi_{R}\right)_{\alpha}^{L} & =\frac{4 \tilde{\beta}_{\alpha}^{R}\left(\tilde{I}_{\alpha}^{R}\right)^{2}\left(\tilde{D}_{\alpha}^{L}-\tilde{\beta}_{\alpha}^{R} \tilde{c}_{\alpha}^{R}\right)^{2}}{\left(6 \tilde{\beta}_{\alpha}^{R} \tilde{I}_{\alpha}^{R}-\left(\tilde{\gamma}_{\alpha}^{L}\right)^{2}\right)^{2}} .
\end{aligned}
$$

Remark 3. If $\alpha=1$, then the parameters $\tilde{c}, \tilde{D}, \tilde{\beta}$, $\tilde{\gamma}$ and $\tilde{I}$ reduce to crisp numbers, then the optimal solutions in Theorems 12 and 13 are

$$
\begin{aligned}
m^{* * *} & =\frac{2 I(D-\beta c)}{6 \beta I-\gamma^{2}} . \\
w^{* * * *} & =\frac{2 I(D+2 \beta c)-\gamma^{2} c}{6 \beta I-\gamma^{2}} . \\
\theta^{* * *} & =\frac{\gamma(D-\beta c)}{6 \beta I-\gamma^{2}} .
\end{aligned}
$$

\section{Numerical Examples}

Because the optimal equilibrium decisions obtained in above Section are in very complicated forms, we have to conduct numerical examples to illustrate the computational process of fuzzy models proposed in this paper. We will also perform the impacts of the fuzziness of parameters $\tilde{\beta}, \tilde{\gamma}$ and the confidence level $\alpha$ on these models.

Consider the case where the retailer orders new greening household appliances from the manufacturer, and then he retails them to the end customers. The data used in the numerical examples are estimated from the Chinese household appliances manufacturing industry. These data have been properly handled before being 
adopted. We think these data can represent the real condition duce to lack of historical data when supply chain participants make their optimal decisions.

The linguistic descriptions and its corresponding triangular fuzzy variables determined by the experiences of experts are shown in Table 1.

Table 1 . The triangular fuzzy variables

\begin{tabular}{lll}
\hline Parameter & Linguistic description & Fuzzy variable \\
\hline$\tilde{D}$ & Large (about 1000) & $(900,1000,1100)$ \\
& Small (about 500) & $(400,500,600)$ \\
$\tilde{\beta}$ & Very sensitive (about 50) & $(45,50,55)$ \\
& Sensitive (about 40) & $(35,40,45)$ \\
$\tilde{\gamma}$ & Very sensitive (about 40) & $(35,40,45)$ \\
& Sensitive (about 30) & $(25,30,35)$ \\
$\tilde{I}$ & Very sensitive (about 30) & $(25,30,35)$ \\
& Sensitive (about 20) & $(15,20,25)$ \\
$\tilde{c}$ & High (about 13) & $(11,13,15)$ \\
& Medium (about 10) & $(9,10,11)$ \\
& Low (about 7) & $(6,7,8)$ \\
\hline
\end{tabular}

\subsection{Discussion 1}

We first assume that the parameter $\tilde{D}$ is large, ( $\tilde{D}$ is about 1000 ), price elastic coefficient $\tilde{\beta}$, greening level elastic coefficient $\tilde{\gamma}$ and investment elastic coefficient $\tilde{I}$ are all in sensitive level, ( $\tilde{\beta}$ is about $40, \tilde{\gamma}$ is about 30 and $\tilde{I}$ is about 20 ), the producing $\operatorname{cost} \tilde{c}$ is medium, ( $\tilde{c}$ is about $10)$. The expected values, $\alpha$-optimistic values and $\alpha$ pessimistic values related to these triangular fuzzy numbers are listed in Table 2.

Table 2. Triangular fuzzy variables, and their expected, $\alpha$ optimistic and $\alpha$-pessimistic values

\begin{tabular}{llll}
\hline \multicolumn{1}{c}{$\begin{array}{c}\text { Triangular fuzzy } \\
\text { variable }\end{array}$} & $\begin{array}{c}\text { Expected } \\
\text { value }\end{array}$ & $\begin{array}{c}\alpha \text {-optimistic } \\
\text { value }\end{array}$ & $\begin{array}{c}\alpha \text {-pessimistic } \\
\text { value }\end{array}$ \\
\hline$\tilde{D}=(900,1000,1100)$ & 100 & $1100-100 \alpha$ & $900+100 \alpha$ \\
$\tilde{\beta}=(35,40,45)$ & 40 & $45-5 \alpha$ & $35+5 \alpha$ \\
$\tilde{\gamma}=(25,30,35)$ & 30 & $35-5 \alpha$ & $25+5 \alpha$ \\
$\tilde{I}=(15,20,25)$ & 20 & $25-5 \alpha$ & $15+5 \alpha$ \\
$\tilde{c}=(9,10,11)$ & 10 & $11-\alpha$ & $9+\alpha$ \\
\hline
\end{tabular}

From Table 2, we have

$$
\begin{aligned}
& E[\tilde{\beta} \tilde{c}]=\frac{1}{2} \int_{0}^{1}\left(\tilde{\beta}_{\alpha}^{L} \tilde{c}_{\alpha}^{L}+\tilde{\beta}_{\alpha}^{R} \tilde{c}_{\alpha}^{R}\right) \mathrm{d} \alpha=\frac{1205}{3}, \\
& \int_{0}^{1}\left(\tilde{\gamma}_{\alpha}^{L} \tilde{c}_{\alpha}^{R}+\tilde{\gamma}_{\alpha}^{R} \tilde{c}_{\alpha}^{L}\right) \mathrm{d} \alpha=\frac{1790}{3}, \\
& \frac{1}{2} \int_{0}^{1}\left(\tilde{D}_{\alpha}^{L} \tilde{c}_{\alpha}^{R}+\tilde{D}_{\alpha}^{R} \tilde{c}_{\alpha}^{L}\right) \mathrm{d} \alpha=\frac{29900}{3} .
\end{aligned}
$$

The results of the optimal expected solutions under three non-cooperative games are showed in Tables 3 and 4.

Table 3. Expected values of the equilibrium prices under three non-cooperative games

\begin{tabular}{ccccc}
\hline & $m$ & $w$ & $p$ & $\theta$ \\
\hline MS & 4.367 & 18.777 & 23.144 & 3.348 \\
RS & 7.507 & 15.264 & 22.770 & 3.989 \\
VN & 6.159 & 16.201 & 22.360 & 4.692 \\
\hline
\end{tabular}

\begin{tabular}{|c|c|c|c|}
\hline & $E\left[\Pi_{R}\right]$ & $E\left[\Pi_{M}\right]$ & $E\left[\Pi_{S C}\right]$ \\
\hline MS & 762.976 & 1386.471 & 2149.447 \\
\hline $\mathrm{RS}$ & 1567.936 & 859.074 & 2427.010 \\
\hline $\mathrm{VN}$ & 1517.424 & 1165.755 & 2683.179 \\
\hline
\end{tabular}

Table 4. Expected values of the equilibrium profits under three non-cooperative games

From Tables 3 and 4, we can find that

(1)The optimal green innovation's level $\theta$ is the highest in the VN case when the actors have equal bargaining power. The MS case provides the lowest level of green innovation this is because under this case the full costs of greening are afforded by manufacturer.

(2)The wholesale price $w$ under the MS case is the highest, followed by $\mathrm{VN}$ and then RS cases, this is because that the manufacturer incurs green costs and has the dominant power in pricing of the green product. The profit margin of the retailer $m$ is highest in the RS case this is because in this case manufacturer charges a low wholesale price $w$. Under MS case, The retail price $p$ is the highest, followed by RS and then VN cases.

(3)The retailer makes the smallest profit under MS case, and the largest under RS case. On the other hand, the manufacturer's fuzzy expected profits are in the reverse order. It shows that the more power the actor has the more fuzzy expected profits he can derive. That is, the retailer's fuzzy expected profit is the largest when the retailer is the leader, and the manufacturer's largest when the manufacturer is the leader in the channel.

(4)The integrated system obtains his largest expected profit in the $\mathrm{VN}$ case when no actor is a channel leader. However, the manufacturer or the retailer has an incentive to be a leader this is because as a leader he can obtain more fuzzy expected profit.

(5) Under VN case, the retail price $p$ is the lowest, and the level of green innovation $\theta$ is highest. For the customers, this means VN case is a preferred policy. 


\subsection{Discussion 2}

In this subsection, we discuss the impacts of the fuzziness of parameters $\tilde{\beta}$ and $\tilde{\gamma}$ on prices, level of green innovation and expected profits under MS, RS and VN cases. Decreasing the fuzzy degree of the parameters $\tilde{\beta}, \tilde{\gamma}$ and observing their impacts. We use the same values of other parameters as in Discussion 1. Tables 5, 6,7 and 8 give the solutions as follows.

Table 5. The change of the expected value of the prices for three fuzzy models with the fuzzy degree of parameter $\tilde{\beta}$

\begin{tabular}{cccccc}
\hline & $\tilde{\beta}$ & $m$ & $w$ & $p$ & $\theta$ \\
\hline \multirow{4}{*}{ MS } & $(35,40,45)$ & 4.367 & 18.777 & 23.144 & 3.348 \\
& $(36,40,44)$ & 4.368 & 18.770 & 23.139 & 3.343 \\
& $(37,40,43)$ & 4.370 & 18.764 & 23.134 & 3.338 \\
& $(38,40,42)$ & 4.371 & 18.758 & 23.128 & 3.332 \\
& $(39,40,41)$ & 4.372 & 18.751 & 23.123 & 3.327 \\
RS & $(35,40,45)$ & 7.507 & 15.264 & 22.770 & 3.989 \\
& $(36,40,44)$ & 7.508 & 15.257 & 22.765 & 3.984 \\
& $(37,40,43)$ & 7.510 & 15.249 & 22.760 & 3.979 \\
& $(38,40,42)$ & 7.512 & 15.242 & 22.754 & 3.973 \\
& $(39,40,41)$ & 7.514 & 15.235 & 22.749 & 3.968 \\
VN & $35,40,45)$ & 6.159 & 16.201 & 22.360 & 4.692 \\
& $(36,40,44)$ & 6.161 & 16.194 & 22.355 & 4.687 \\
& $(37,40,43)$ & 6.162 & 16.187 & 22.349 & 4.682 \\
& $(38,40,42)$ & 6.164 & 16.180 & 22.344 & 4.677 \\
& $(39,40,41)$ & 6.165 & 16.174 & 22.339 & 4.672 \\
\hline
\end{tabular}

Table 6. The change of the expected value of the profits for three fuzzy models with the fuzzy degree of parameter $\tilde{\beta}$

\begin{tabular}{lcccc}
\hline & $\tilde{\beta}$ & $E\left[\Pi_{R}\right]$ & $E\left[\Pi_{M}\right]$ & $E\left[\Pi_{S C}\right]$ \\
\hline MS & $(35,40,45)$ & 762.976 & 1386.471 & 2149.447 \\
& $(36,40,44)$ & 763.346 & 1378.757 & 2142.104 \\
& $(37,40,43)$ & 763.717 & 1371.045 & 2134.762 \\
& $(38,40,42)$ & 764.088 & 1363.335 & 2127.423 \\
& $(39,40,41)$ & 764.459 & 1355.626 & 2120.085 \\
RS & $(35,40,45)$ & 1567.936 & 859.074 & 2427.010 \\
& $(36,40,44)$ & 1568.698 & 851.104 & 2419.802 \\
& $(37,40,43)$ & 1569.459 & 843.136 & 2412.595 \\
& $(38,40,42)$ & 1570.221 & 835.169 & 2405.391 \\
& $(39,40,41)$ & 1570.984 & 827.204 & 2398.188 \\
VN & $35,40,45)$ & 1517.424 & 1165.755 & 2683.179 \\
& $(36,40,44)$ & 1518.161 & 1157.934 & 2676.095 \\
& $(37,40,43)$ & 1518.898 & 1150.114 & 2669.013 \\
& $(38,40,42)$ & 1519.636 & 1142.297 & 2661.933 \\
& $(39,40,41)$ & 1520.373 & 1134.481 & 2654.854 \\
\hline
\end{tabular}

Table 7. The change of the expected value of the prices for three fuzzy models with the fuzzy degree of parameter $\tilde{\gamma}$

\begin{tabular}{cccccc}
\hline & $\tilde{\gamma}$ & $m$ & $w$ & $p$ & $\theta$ \\
\hline \multirow{2}{*}{ MS } & $(25,30,35)$ & 4.367 & 18.777 & 23.144 & 3.348 \\
& $(26,30,34)$ & 4.366 & 18.773 & 23.138 & 3.339 \\
& $(27,30,33)$ & 4.364 & 18.769 & 23.133 & 3.329 \\
& $(28,30,32)$ & 4.362 & 18.766 & 23.128 & 3.319 \\
& $(29,30,31)$ & 4.360 & 18.762 & 23.122 & 3.310 \\
RS & $(25,30,35)$ & 7.507 & 15.264 & 22.770 & 3.989 \\
& $(26,30,34)$ & 7.503 & 15.261 & 22.765 & 3.979 \\
& $(27,30,33)$ & 7.500 & 15.259 & 22.760 & 3.969 \\
& $(28,30,32)$ & 7.497 & 15.257 & 22.754 & 3.959 \\
& $(29,30,31)$ & 7.494 & 15.255 & 22.749 & 3.950 \\
VN & $(25,30,35)$ & 6.159 & 16.201 & 22.360 & 4.692 \\
& $(26,30,34)$ & 6.157 & 16.198 & 22.355 & 4.682 \\
& $(27,30,33)$ & 6.154 & 16.196 & 22.350 & 4.672 \\
& $(28,30,32)$ & 6.151 & 16.193 & 22.345 & 4.662 \\
& $(29,30,31)$ & 6.149 & 16.191 & 22.340 & 4.651 \\
\hline
\end{tabular}

Table 8. The change of the expected value of the profit for three fuzzy models with the fuzzy degree of parameter $\tilde{\gamma}$

\begin{tabular}{ccccc}
\hline & $\tilde{\gamma}$ & $E\left[\Pi_{R}\right]$ & $E\left[\Pi_{M}\right]$ & $E\left[\Pi_{S C}\right]$ \\
\hline MS & $(25,30,35)$ & 762.976 & 1386.471 & 2149.447 \\
& $(26,30,34)$ & 762.341 & 1385.356 & 2147.697 \\
& $(27,30,33)$ & 761.706 & 1384.245 & 2145.951 \\
& $(28,30,32)$ & 761.071 & 1383.137 & 2144.208 \\
& $(29,30,31)$ & 760.437 & 1382.032 & 2142.469 \\
RS & $(25,30,35)$ & 1567.936 & 859.074 & 2427.010 \\
& $(26,30,34)$ & 1566.631 & 858.399 & 2425.030 \\
& $(27,30,33)$ & 1565.326 & 857.726 & 2423.052 \\
& $(28,30,32)$ & 1564.022 & 857.057 & 2421.079 \\
& $(29,30,31)$ & 1562.718 & 856.391 & 2419.109 \\
VN & $(25,30,35)$ & 1517.424 & 1165.755 & 2412.595 \\
& $(26,30,34)$ & 1516.161 & 1164.824 & 2680.985 \\
& $(27,30,33)$ & 1514.898 & 1163.896 & 2678.794 \\
& $(28,30,32)$ & 1513.636 & 1162.972 & 2676.608 \\
& $(29,30,31)$ & 1512.374 & 1162.050 & 2674.425 \\
\hline
\end{tabular}

From Tables 5, 6, 7 and 8, we can find that

(6)When the fuzziness of the parameter $\tilde{\beta}$ decreases, the profit margin $m$ and expected profit of retailer increase slightly in the three game cases. Therefore, the retailer should seek as low fuzziness of parameter $\tilde{\beta}$ as possible.

(7)The wholesale price $w$, retail price $p$, level of green innovation $\theta$, expected profit for manufacturer and whole 
supply chain drop slightly in three cases, as the fuzziness of parameter $\tilde{\beta}$ falls.

(8)When the fuzziness of the parameter $\tilde{\gamma}$ decreases, the profit margin $m$, prices $w$ and $p$, level of green innovation $\theta$ and profits for supply chain members will all increase.

\subsection{Discussion 3}

Thirdly, we present the results of the $\alpha$-optimistic values and $\alpha$-pessimistic values under three decentralized channel decisions shown in Tables 9, 10, 11 and 12, respectively. In Tables 9, 10, 11 and 12, the 2th, 8th and 14 th rows show the optimal equilibrium decisions under three cases in crisp environment at $\alpha=1$.

Table 9. $\alpha$-optimistic value of the equilibrium prices under three cases with respect to $\alpha$

\begin{tabular}{cccccc}
\hline & $\alpha$ & $m$ & $w$ & $p$ & $\theta$ \\
\hline \multirow{4}{*}{ MS } & 1.00 & 4.364 & 18.727 & 23.091 & 3.273 \\
& 0.95 & 4.487 & 18.924 & 23.411 & 3.436 \\
& 0.90 & 4.614 & 19.128 & 23.742 & 3.608 \\
& 0.85 & 4.745 & 19.339 & 24.084 & 3.790 \\
& 0.80 & 4.879 & 19.559 & 24.438 & 3.981 \\
$\mathrm{RS}$ & 0.75 & 5.019 & 19.787 & 24.806 & 4.182 \\
& 1.00 & 7.500 & 15.217 & 22.717 & 3.913 \\
& 0.95 & 7.667 & 15.360 & 23.026 & 4.142 \\
& 0.90 & 7.835 & 15.512 & 23.347 & 4.389 \\
& 0.85 & 8.005 & 15.675 & 23.680 & 4.652 \\
$\mathrm{VN}$ & 0.80 & 8.177 & 15.850 & 24.027 & 4.936 \\
& 0.75 & 8.351 & 16.038 & 24.389 & 5.240 \\
& 1.00 & 6.154 & 16.154 & 22.308 & 4.615 \\
& 0.95 & 6.343 & 16.293 & 22.637 & 4.858 \\
& 0.90 & 6.540 & 16.440 & 22.979 & 5.114 \\
& 0.85 & 6.743 & 16.593 & 23.336 & 5.386 \\
& 0.80 & 6.954 & 16.754 & 23.709 & 5.673 \\
& 0.75 & 7.174 & 16.924 & 24.099 & 5.979 \\
\hline
\end{tabular}

Table 10. $\alpha$-optimistic value of the equilibrium profits under three cases with respect to $\alpha$

\begin{tabular}{ccccc}
\hline & $\alpha$ & $\left(\Pi_{R}\right)_{\alpha}^{R}$ & $\left(\Pi_{M}\right)_{\alpha}^{R}$ & $\left(\Pi_{S C}\right)_{\alpha}^{R}$ \\
\hline MS & 1.00 & 761.653 & 1309.091 & 2070.744 \\
& 0.95 & 800.296 & 1367.387 & 2167.683 \\
& 0.90 & 840.898 & 1427.903 & 2268.801 \\
& 0.85 & 883.602 & 1490.754 & 2374.357 \\
& 0.80 & 928.565 & 1556.068 & 2484.633 \\
$\mathrm{RS}$ & 0.75 & 975.963 & 1623.981 & 2599.943 \\
& 1.00 & 1565.217 & 782.609 & 2347.826 \\
& 0.95 & 1648.542 & 824.271 & 2472.713 \\
& 0.90 & 1736.704 & 868.352 & 2605.056 \\
$\mathrm{VN}$ & 0.85 & 1830.141 & 915.071 & 2745.212 \\
& 0.80 & 1929.353 & 964.677 & 2894.029 \\
& 0.75 & 2034.907 & 1017.453 & 3052.360 \\
& 1.00 & 1514.793 & 1088.757 & 2603.550 \\
& 0.95 & 1599.432 & 1133.361 & 2732.793 \\
& 0.90 & 1689.237 & 1179.204 & 2868.440 \\
& 0.85 & 1784.665 & 1226.302 & 3010.967 \\
& 0.80 & 1886.233 & 1274.671 & 3160.903 \\
& 0.75 & 1994.521 & 1324.319 & 3318.840 \\
\hline
\end{tabular}

Table 11. $\alpha$-pessimistic value of the equilibrium prices under

\begin{tabular}{cccccc}
\multicolumn{6}{c}{ three cases with respect to $\alpha$} \\
\hline \multirow{2}{*}{ MS } & $\alpha$ & $m$ & $w$ & $p$ & $\theta$ \\
& 1.00 & 4.364 & 18.727 & 23.091 & 3.273 \\
& 0.95 & 4.244 & 18.537 & 22.781 & 3.117 \\
& 0.90 & 4.127 & 18.354 & 22.480 & 2.969 \\
& 0.85 & 4.103 & 18.176 & 22.189 & 2.828 \\
$\mathrm{RS}$ & 0.80 & 3.902 & 18.004 & 21.906 & 2.694 \\
& 0.75 & 3.794 & 17.838 & 21.631 & 2.566 \\
& 1.00 & 7.500 & 15.217 & 22.717 & 3.913 \\
& 0.95 & 7.335 & 15.084 & 22.420 & 3.698 \\
& 0.90 & 7.172 & 14.960 & 22.132 & 3.496 \\
$\mathrm{VN}$ & 0.85 & 7.011 & 14.842 & 21.853 & 3.307 \\
& 0.80 & 6.851 & 14.732 & 21.584 & 3.130 \\
& 0.75 & 6.693 & 14.629 & 21.322 & 2.962 \\
& 1.00 & 6.154 & 16.154 & 22.308 & 4.615 \\
& 0.95 & 5.971 & 16.021 & 21.992 & 4.386 \\
& 0.90 & 5.794 & 15.894 & 21.687 & 4.169 \\
& 0.85 & 5.622 & 15.772 & 21.394 & 3.963 \\
& 0.80 & 5.456 & 15.656 & 21.111 & 3.767 \\
& 0.75 & 5.294 & 15.544 & 20.838 & 3.581 \\
\hline
\end{tabular}

Table 12. $\alpha$-pessimistic value of the equilibrium profits under three cases with respect to $\alpha$

\begin{tabular}{ccccc}
\hline & $\alpha$ & $\left(\Pi_{R}\right)_{\alpha}^{L}$ & $\left(\Pi_{M}\right)_{\alpha}^{L}$ & $\left(\Pi_{S C}\right)_{\alpha}^{L}$ \\
\hline \multirow{2}{*}{ MS } & 1.00 & 761.653 & 1309.091 & 2070.744 \\
& 0.95 & 724.840 & 1252.907 & 1977.747 \\
& 0.90 & 689.740 & 1198.737 & 1888.477 \\
& 0.85 & 656.246 & 1146.490 & 1802.735 \\
& 0.80 & 624.261 & 1096.081 & 1720.342 \\
$\mathrm{RS}$ & 0.75 & 593.696 & 1047.434 & 1641.129 \\
& 1.00 & 1565.217 & 782.609 & 2347.826 \\
& 0.95 & 1486.341 & 743.171 & 2229.512 \\
& 0.90 & 1411.570 & 705.785 & 2117.355 \\
$\mathrm{VN}$ & 0.85 & 1340.596 & 670.298 & 2010.895 \\
& 0.80 & 1273.147 & 636.574 & 1909.721 \\
& 0.75 & 1208.977 & 604.488 & 1813.465 \\
& 1.00 & 1514.793 & 1088.757 & 2603.550 \\
& 0.95 & 1434.910 & 1045.374 & 2480.328 \\
& 0.90 & 1359.416 & 1003.189 & 2362.605 \\
& 0.85 & 1287.985 & 962.181 & 2250.165 \\
& 0.80 & 1220.320 & 922.327 & 2142.646 \\
& 0.75 & 1156.155 & 883.604 & 2039.759 \\
\hline
\end{tabular}

From Tables 9, 10, 11 and 12, we can find that

(9) In the three different fuzzy models, the $\alpha$-optimistic values of profit margin, prices, level of green innovation and profits for supply chain participants increase, as the confidence level $\alpha$ decreases. That is to say, if the confidence level of the supply chain participant's profits $\alpha=1$, then the supply chain participants derive their smallest profits. It means that the less responsible of supply chain participants to risk, the lower profits will be when they are risk preferable.

(10) In the three different fuzzy models, the $\alpha$ pessimistic values of profit margin, prices, greening level and profits for supply chain actors increase, as the confidence level $\alpha$ increases. That is to say, if the confidence level of the supply chain participant's profits 
$\alpha=1$, then the supply chain participants derive their largest profits. It means that the more responsible of supply chain participants to risk, the larger profits will be when they are risk averse.

In practice, the supply chain participants can alter the value of the parameter $\alpha$ to obtain the different equilibrium decisions under the different level of the supply chain participant's profits. The equilibrium decision reflects the different risk attitude of the supply chain participant to market demand uncertainty and different prediction of possibility level.

(11) It is interesting to compare the solutions of this paper with the work of Ghosh and Shah ${ }^{3}$. When $\alpha=1$, the solutions in this study will be the conventional solutions according to the method of Ghosh and Shah ${ }^{3}$. In Tables9, 10, 11 and 12, the 2th, 8th and 14th rows show the optimal equilibrium decisions under three cases in crisp environment. Compared these optimal equilibrium decisions in crisp environment to those in fuzzy decision making environment showed in Table 3, we observe that our profit margin, wholesale price, retail price and level of green innovation are all higher than those in crisp results. The manufacturer's profit is lower, while the retailer's profit and supply chain system's profit are higher when they face fuzzy uncertainty. It means that the retailer could benefit from the fuzzy environment, while the manufacturer could suffer from this environment.

\section{Conclusions}

This paper deals with the coordination strategy in a $\mathrm{m}$ In this paper, we considered three different fuzzy models in green supply chain, in which supply chain participants pursued three different power balance scenarios. For each model, we provided the optimal equilibrium decisions of the manufacturer and the retailer when they were risk neutral, risk preferable and risk averse. We also found that under certain circumstances, the formulated fuzzy supply chain models can degenerate into crisp models. Besides, we show that the different power structures, the confidence level of the supply chain participant's profits and fuzzy degree of parameters affect the final optimal solutions.

The current fuzzy green supply chain models have some limitations. The fuzzy demand for supply chain is assumed as a linear function. Another limitation is that we only consider one manufacturer and one retailer in GSC. Further research can focus on analyzing some more complicated greening policies in which some other kinds of demand functions or multiple supply chain participants exist. Still, we will develop the channel coordination in GSC under a fuzzy decision environment.

\section{Acknowledgements}

This work was supported by the Shandong Provincial Natural Science Foundation, China (No. ZR2015GQ 001), and the Project of Shandong Provincial Higher Educational Humanity and Social Science Research Program (No. J15WB04).

\section{References}

1. J.B. Sheu, Green supply chain management, reverse logistics and nuclear power generation, Transpor Res ELog. 44(1) (2008) 19-46.

2. J.B. Sheu, Bargaining framework for competitive green supply chains under governmental financial intervention, Transpor Re. E-Log. 47(5) (2011) 573-592.

3. G. Ghosh amd J. Shah, A comparative analysis of greening policies across supply chain structures, Int J Prod Econ. 135(2) (2012) 568-583.

4. G. Ghosh and J. Shah, Supply chain analysis under green sensitive consumer demand and cost sharing contract, Int Prod Econ. 164(2015) 319-329.

5. S. Barari, G. Agarwal, W.J. Zhang, B. Mahanty and M.K. Tiwari, A decision framework for the analysis of green supply chain contracts: An evolutionary game approach, Expert Syst Appl. 39(3) (2012) 2965-2976.

6. S. Swami and J. Shah, Channel coordination in green supply chain management: the case of package size and shelf-space allocation, Technol Ope Manag. 2 (1) (2011) $50-59$.

7. S. Swami and J. Shah, Channel coordination in green supply chain management, J Oper Res Soc. 64(3) (2013) 336-351.

8. S.M.J. Mirzapour Al-e-hashem, A. Baboli and Z. Sazvar, A stochastic aggregate production planning model in a green supply chain: Considering flexible lead times, nonlinear purchase and shortage cost functions, Eur J Ope Res. 230 (1) (2013) 26-41.

9. L. Tomasin, G.M. Pereira, M. Borchardt and M.A. Sellitto, How can the sales of green products in the Brazilian supply chain be increased? J Clean Prod. 47(2013) 274-282.

10. C.T. Zhang and L.P. Liu, Research on coordination mechanism in three-level green supply chain under noncooperative game, Appl Math Model. 37(5) (2013) 33693379.

11. R.N. Xu and X.Y. Zhai, Optimal models for single-period supply chain problems with fuzzy demand, Inf Sci. 178(17) (2008) 3374-3381. 
12. R.N. Xu and X.Y. Zhai, Analysis of supply chain coordination under fuzzy demand in a two-stage supply chain, Appl Math Model. 34(1) (2010) 129-139.

13. C. Zhou, R. Zhao and W. Tang, Two-echelon supply chain games in a fuzzy environment, Comput Ind Eng. 55(2) (2008) 390-405.

14. Y. Yu and T. Jin, The return policy model with fuzzy demands and asymmetric information, Appl Soft Comput. 11(2) (2011) 1669-1678.

15. J. Wei and J. Zhao, Pricing decisions with retail competition in a fuzzy closed-loop supply chain, Expert Syst Appl. 38(9) (2011) 11209-11216.

16. F. Ye and Y.N. Li, A Stackelberg single-period supply chain inventory model with weighted possibilistic mean values under fuzzy environment, Appl Soft Comput. 11(8) (2011) 5519-5527.

17. J. Zhao, W. Tang and J. Wei, Pricing decision for substitutable products with retail competition in a fuzzy environment, Int J Prod Econ. 135 (1) (2012) 144-153.

18. J. Zhao, W. Tang, R. Zhao and J. Wei, Pricing decisions for substitutable products with a common retailer in fuzzy environments, Eur J Ope Res. 216(2) (2012) 409-419.

19. J. Wei and J. Zhao, Reverse channel decisions for a fuzzy closed-loop supply chain, Appl Math Model. 37(3) (2013) 1502-1513.

20. J. Zhao and L. Wang, Pricing and retail service decisions in fuzzy uncertainty environments, Appl Math Comput. 250 (2015) 580-592.
21. Y. Yu, J. Zhu and C. Wang, A newsvendor model with fuzzy price-dependent demand, Appl Math Model. 37(5) (2013) 2644-2661.

22. S. Sang, Revenue sharing contract in a multi-echelon supply chain with fuzzy demand and asymmetric information, Int J Comput Int Sys. 9 (6) (2016) 1028-1040.

23. S.Y. Chang and T.Y. Yeh, A two-echelon supply chain of a returnable product with fuzzy demand, Appl Math Model. 37(6) (2013) 4305-4315.

24. A.A. Khamseh, F. Soleimani and B. Naderi, Pricing decisions for complementary products with firm's different market powers in fuzzy environments, J Intell Fuzzy Syst. 27(5) (2014) 2327-2340.

25. S. Sang, Price competition of manufacturers in supply chain under a fuzzy decision environment, Fuzzy Optim Decis Making. 14(3) (2015) 335-363.

26. S. Nahmias, Fuzzy variables. Fuzzy Set Syst. 1(2) (1978) 97-110.

27. B. Liu and Y. Liu, Expected value of fuzzy variable and fuzzy expected value model. IEEE T Fuzzy Syst. 10(4) (2002) 445-450.

28. R. Zhao, W. Tang and H. Yun, Random fuzzy Renewal process. Eur J Ope Res. 169(1) (2006) 189-201.

29. B. Liu and K. Iwamura, Chance constrained programming with fuzzy parameters, Fuzzy Set Syst. 94(2) (1998) 227237.

30. B. Liu and K. Iwamura, A note on chance constrained programming with fuzzy coefficients, Fuzzy Set Syst. 100(1-3) (1998) 229-233. 